\title{
Transient Response of Structural Dynamic Systems with Parametric Uncertainty
}

\author{
Abhishek Kundu ${ }^{1}$ and Sondipon Adhikari ${ }^{2}$
}

${ }^{1}$ Dept. of Aerospace Engineering, College of Engineering, Swansea Univ., Singleton Park SA2 8PP, U.K. (corresponding author). E-mail: a.kundu.577613@swansea.ac.uk

2

Note. This manuscript was submitted on August 6, 2012; approved on March 22, 2013; published online on April 1, 2013. Discussion period open until July 1, 2014; separate discussions must be submitted for individual papers. This paper is part of the Journal of Engineering Mechanics, Vol. 140, No. 2, February 1, 2014. CASCE, ISSN 0733-9399/2014/ 2-0001-0017/\$25.00. (e.g., perturbation methods, Neumann expansion method, and some Galerkin-type method using a polynomial expansion technique).

Various MCS technique approaches, which are nonintrusive in $\mathbf{4}$ nature, have been analyzed and used in the context of structural dynamics problems (Schuëller 2001; Hurtado and Barbat 1998; Pradlwarter and Schuëller 1997). The advantage of nonintrusive techniques, such as the MCS in conjunction with advanced interpolation schemes, lies in the fact that they only make use of the simple deterministic codes to solve the stochastic problem at the sample points and hence do not require any further development. Also, they are naturally suited for parallelization. The convergence of the MCS, however, is slow, and its computational cost grows as a polynomial with the dimension of the input stochastic space. The computational efficacy of these sample-based techniques can be substantially improved by reducing the problem to important random variables using the principle component analysis (Schuëller 2001) and with various efficient sampling techniques, such as importance sampling, multipoint estimate method, stratified sampling, Latin hypercube sampling, orthogonal sampling, which can be classified under the variance reduction techniques (Yamazaki and Shinozuka 1988; Schuëller et al. 1991), and the response surface method (Bucher and Bourgund 1990) or metamodeling [e.g., Kriging metamodel (Kleijnen 2009)]. However, the limitations of these techniques are dictated by the input stochastic space dimension. Uncertain structural systems represented by few random variables subjected to deterministic loading can be well suited to variance reduction procedures.

Nonstatistical approaches can be based on a perturbation method (Kleiber and Hien 1992; Falsone and Impollonia 2002), or equivalently the lower-order Taylor approximation and Neumann expansion method (Yamazaki et al. 1988; Zhu et al. 1992), all of which come down to the estimation of the response surface in a parameter space. On the other hand, the Galerkin-type methods (Deb et al. 2001; Babuska et al. 2005; Matthies and Keese 2005), developed with differing choice of the approximation space, systematically lead to a high-precision solution, allowing the response to be expressed explicitly in terms of the basic random variables describing 
the uncertainties. Their principle drawback lies in the fact that the dimensionality of the resulting system of linear equations is high. The Wiener-Hermite expansions, in conjunction with the FEMs, have been widely applied to different problems (Pettit and Beran 2006; Ghanem and Spanos 1991). It was extended to generalized polynomial chaos (gPC) (Xiu and Karniadakis 2002), which provided an optimal convergence of the solution using the so-called Askey scheme.

In this paper, the authors review the problem of unsteady (transient) dynamics of structural systems and propose an alternative method by projecting the solution in a preconditioned stochastic Krylov space. The solution is approximated in a stochastic subspace using spectral functions of different orders, which are highly nonlinear functions of the input random variables. The statistical properties of the response quantities change with time, and the authors investigate the time evolution of this uncertainty propagation with different solution techniques considered herein. The objective here is to tackle the problem of the growing dimensionality of the classical spectral Galerkin approach for long time integration with an alternative formulation of the spectral function approach.

A damped structural dynamic system with stochastic parameters, defined on domain $\mathcal{D}$, subjected to an externally applied force excitation $p$ varying with time $t$ is considered. The force equilibrium condition gives the following stochastic partial differential equation (SPDE):

$$
\rho \frac{\partial^{2} u}{\partial t^{2}}+\mathcal{L}_{\eta} \frac{\partial u}{\partial t}+\operatorname{div}\left(\sigma_{\alpha}(u)\right)=p \quad \text { on } \mathcal{D}
$$

with the associated Dirichlet boundary condition

$$
u=0 ; \quad \text { on } \partial \mathcal{D}
$$

where $\sigma_{\alpha}(u)=$ stress related to the displacement field $u=\{u: u(\mathbf{r}, t ; \theta)$ $\in \mathcal{D} \times T \times \Theta\}, \rho=$ mass density, and $p=$ external volume force density varying with time $t \in T \rightarrow \mathbb{R} . \mathcal{L}_{\eta}$ is the damping operator, with $\eta$ as the damping parameter, and it can be used to represent different damping models, such as the strain rate-dependent viscous damping or the velocity-dependent viscous damping. Also, $\mathcal{D} \in \mathbb{R}^{d}$ is a bounded domain with piecewise Lipschitz boundary $\delta \mathcal{D}$, where $d \leq 3$ is the spatial dimension and $T \in \mathbb{R}^{+}$is the time. $(\Theta, \mathcal{F}, P)$ is the probability space where $\theta \in \Theta$ is a sample point from the sampling space $\Theta, \mathcal{F}$ is the associated Borel $\sigma$-algebra, and $P$ is the image probability measure. The constitutive equations relating the stress field to the displacement $u$ are given as

$$
\sigma_{\alpha}(u)=\alpha(\mathbf{r}, \theta): \varepsilon(u)
$$

where $\alpha=$ Hooke's elasticity tensor and is a second-order, stationary, square integrable random field such that $\alpha: \mathbb{R}^{d} \times \Theta \rightarrow \mathbb{R}$. Depending on the physical problem, the random field $\alpha(\mathbf{r}, \theta)$ can be used to model different physical systems. Here, $p$ denotes the timedependent deterministic excitation field for which the solution $u$ is sought in the time domain.

The paper is organized as follows: the next section gives the details of the finite-element (FE) implementation of the SPDE in the time domain, which includes the discretization techniques of the stochastic field with a finite number of random variables. The description of the stochastic system matrices is also included in this section. After this, the details of the solution techniques of the discretized stochastic FE system using the spectral function method in the stochastic subspace are given. In the section following, the numerical implementation of the proposed solution technique for the case of a cantilever Euler-Bernoulli beam under an impulse load is discussed. This section includes a comparison of the time-domain response of the beam using different solution techniques, such as the direct MCS, the proposed spectral function approach, and the fourth-order polynomial chaos (PC) approach, which demonstrate the accuracy and computational efficacy of the proposed methodology. A detailed discussion of a posteriori error analysis and the behavior of the autocorrelation function (ACF) has also been included. The final section lists the significant conclusions that can be reached from this study.

\section{FE Modeling of the SPDE}

\section{Discretization of the Parametric Random Field}

The stochastic parameter used as an input to the mathematical model is defined on a compact region on $\mathbb{R}^{d}$ and a probability space $(\Theta, \mathcal{F}$, $P$ ), and is represented as $a(\mathbf{r}, \theta): \mathcal{D} \times \Theta$. This parameter is written as a series expansion in a variable separable form as

$$
a(\mathbf{r}, \theta)=\sum_{i} \varphi_{i}(\mathbf{r}) \kappa(\theta)=\boldsymbol{\varphi}(\mathbf{r}) \mathcal{K}(\theta)
$$

where the vector of random functions $\mathcal{K}(\theta)=\left\{\kappa_{1}(\theta), \ldots, \kappa_{m}(\theta)\right\}^{T}$ [ is weighted by the spatial shape functions $\varphi(\mathbf{r})=\left\{\varphi_{1}(\mathbf{r}), \ldots\right.$, $\left.\varphi_{m}(\mathbf{r})\right\}$. These shape functions can be chosen based on the type of series representation used to represent the random field, which includes (1) the interpolation method (Liu et al. 1986), (2) the midpoint method ( $\mathrm{Li}$ and Kiureghian 1993), (3) the expansion optimal linear estimation (EOLE) method (Li and Kiureghian 1993), (4) the spatial average method (Vanmarcke 1983), (5) the orthogonal expansion method, and finally, and perhaps most importantly, (6) the spectral decomposition of the stochastic process, which relies on discretizing the latter with a finite number of spectral components of the covariance kernel associated with the random process.

The objective of the spectral decomposition method is to express the random parameter with a set of a denumerable number of orthogonal random variables (spanning the stochastic Hilbert space) and the associated spatial eigenfunctions. Let $\mathcal{C}_{a}: \mathbb{R}^{d} \times \mathbb{R}^{d} \rightarrow \mathbb{R}$ be a kernel function that admits to the following decomposition:

$$
\int_{\mathcal{D}} C_{a}\left(\mathbf{r}_{1}, \mathbf{r}_{2}\right) \varphi_{j}\left(\mathbf{r}_{1}\right) d \mathbf{r}_{1}=\nu_{j} \varphi_{j}\left(\mathbf{r}_{2}\right), \quad \forall j=1,2, \ldots
$$

Eq. (4) is a homogeneous Fredholm integral equation of the second kind. The function $\mathcal{C}_{a} \varphi$ is defined such that

$$
\left(\mathcal{C}_{a} \varphi\right)\left(\mathbf{r}_{1}\right)=\int_{\mathcal{D}} C_{a}\left(\mathbf{r}_{1}, \mathbf{r}_{2}\right) \varphi\left(\mathbf{r}_{2}\right) d \mathbf{r}_{2} \mathbf{r}_{1}, \mathbf{r}_{2} \in \mathbb{R}^{d}
$$

It can be easily verified that $\mathcal{C}_{a}: L^{2}\left(\mathbb{R}^{d}\right) \rightarrow L^{2}\left(\mathbb{R}^{d}\right)$ is a linear operator on a vector space; hence, Eq. (4) can be expressed as

$$
\mathcal{C}_{a} \varphi=\nu \varphi
$$

A nontrivial solution to this homogeneous equation exists only for those values of $\nu$, which makes $\left(I-\nu \mathcal{C}_{a}\right)$ noninvertible, where $I$ is the identity operator. The covariance functions $\mathcal{C}_{a}$ commonly encountered in the study of randomly parameterized engineering systems are bounded and symmetric; hence, the associated linear operator $\mathcal{C}_{a}$ is compact and self-adjoint. Considering the solution of Eq. (4) lies in some normed vector space, it is possible to represent the random parameter using a finite number of dominant components based on the eigenvalue problem in Eq. (6). 
The Karhunen-Loève (KL) expansion is a special case of this spectral representation of the random field using orthogonal eigencomponents of the covariance kernel. The random parameter $a(\mathbf{r}, \theta): \mathcal{D} \times \Theta$ in the truncated spectral representation takes the form

$$
a(\mathbf{r}, \theta)=a_{0}(\mathbf{r})+\sum_{i=1}^{m} \sqrt{\nu_{i}} \xi_{i}(\theta) \varphi_{i}(\mathbf{r})
$$

where $a_{0}(\mathbf{r})=\mathbb{E}[a(\mathbf{r}, q)]$ is the mean of the stochastic parameter, and $\xi_{i}(\theta)$ are mutually uncorrelated random variables with zero mean $\left\{\mathbb{E}\left[\xi_{i}(q)\right]=0\right\}$ and unit variance $\left\{\mathbb{E}\left[\xi_{i}(\theta)^{2}\right]=1\right\}$. In addition, $\nu_{i}$ and $\varphi_{i}(\mathbf{r})$ are eigenvalues and eigenfunctions satisfying the integral equation [Eq. (4)]. For Gaussian random fields, the $\xi_{i}(\theta)$ are uncorrelated Gaussian random variables by virtue of the property of Gaussian variables. However, the Gaussian random field model is not applicable for strictly positive quantities arising in many practical problems.

Alternatively, when $a(\mathbf{r}, \theta)$ is a non-Gaussian random field, it can be expressed in a mean-square convergent series using the WienerAskey chaos expansion scheme (Xiu and Karniadakis 2002, 2003b; Wan and Karniadakis 2006). To overcome the computational and experimental challenges that may be involved as a result of the basic random variables not being independent in nature, it is desirable to discretize the stochastic process with a set of independent nonGaussian random variables $\hat{\xi}(\theta)=\left\{\hat{\xi}^{(1)}, \ldots, \hat{\xi}^{(n)}\right\}$, and express the components of $\hat{\xi}(\theta)$ with a PC type of expansion using a series of normalized finite-order Hermite polynomials $\varphi_{j}\left(\chi_{i}\right)$ as

$$
\hat{\xi}^{(i)}(\theta)=\sum_{j=1}^{\infty} \vartheta_{j}^{i} \varphi_{j}\left(\chi_{i}\right) \approx \sum_{j=1}^{p} \vartheta_{j}^{i} \varphi_{j}\left(\chi_{i}\right)
$$

where $\chi_{i}=$ Gaussian iid random variables; and $\vartheta_{j}^{i}=$ undetermined deterministic coefficients of the problem, which must be such that the sum of their squares always comes to one.

The formulation presented is applicable to this kind of general decomposition of the random field. For the numerical implementation of the aforementioned method, the probabilistic content of the problem is represented using a finite set of random variables $\boldsymbol{\xi}=\left(\xi_{1}, \xi_{2}, \ldots, \xi_{m}\right): \Theta \rightarrow \mathbb{R}^{m}$. The stochastic problem can be equivalently formulated on the finite dimensional probability space $\left[\Theta^{(m)}, \mathcal{F}^{(m)}, P^{(m)}\right]$, where $\Theta^{(m)}=\operatorname{range}(\boldsymbol{\xi})$ is a subset of $\mathbb{R}^{m}, \mathcal{F}^{(m)}$ is the associated Borel $\sigma$-algebra, and $P^{(m)}$ is the image probability measure.

\section{FE Modeling of the Discretized Random System}

The FE treatment of the governing SPDE involves spatial discretization of the continuum $\mathcal{D} \in \mathbb{R}^{d}$ into domains with polygonal boundaries $\mathcal{D}^{h}$, where $h$ is the mesh-space parameter. Also, it is known from the Doob-Dynkin lemma (Bobrowski 2005), that for the parameterized equation in Eq. (1), where the input randomness is expressed in terms of a finite dimensional vector $\boldsymbol{\xi}(\theta)$ [as in Eq. (7)], the solution can be expressed entirely in terms of the same random variables. The solution of the discretized FE system lies in the Hilbert space $\mathcal{H}\left(\mathcal{D}^{h} \times T \times \Theta\right)$. This space can be expressed in a separable form with the Hilbert spaces $\mathcal{H}_{1}$ and $\mathcal{H}_{2}$ such that $\mathcal{H} \simeq \mathcal{H}_{1} \otimes \mathcal{H}_{2}$. Now, $\mathcal{H}_{1}$ and $\mathcal{H}_{2}$ can be chosen to have different forms, and the solution separable spaces vary accordingly. The FE shape functions are taken to lie in $L_{2}\left(\mathcal{D}^{h}\right)$ space. Strictly speaking, the space of these ansatz functions is governed by the continuity requirement of the field over the element domain; for example, if symmetric bilinear forms are obtained from applying the variational principle to elliptic differential operators of order $2 k$ (and with
Dirichlet boundary conditions), then these trial functions lie in the Hilbert space $H_{0}^{k} \subset L^{2}\left(\mathcal{D}^{h}\right)$ and are $C^{k}$ continuous within the element domain. The principle of virtual work is utilized to arrive at the discretized set of linear algebraic equations from Eq. (1). For the virtual displacements, a set of stochastic kinematically admissible displacement functions $v(\cdot ; \theta)$ satisfying the boundary condition $\left.v(\cdot ; \theta)\right|_{\delta_{D}}=0$ and which have finite strain energy on the spatial domain lying in $E(\mathcal{D}) \subset L^{2}(\mathcal{D})$ is chosen. Denoting the set of all such functions by $E(\mathcal{D})$ gets

$$
\stackrel{o}{E}(\mathcal{D})=\left\{v(\cdot ; \theta): v(\cdot ; \theta) \in E(\mathcal{D}), \quad \text { and }\left.\quad v(\cdot ; \theta)\right|_{\partial \mathcal{D}}=0\right\}
$$

From the principle of virtual work

$$
\int_{\mathcal{D}} v:\left\{\rho \frac{\partial^{2} u}{\partial t^{2}}+\mathcal{L}_{\eta} \frac{\partial u}{\partial t}+\operatorname{div}\left(\sigma_{a}(u)\right\} d \mathbf{r} \quad \forall v \in \stackrel{o}{E}(\mathcal{D})\right.
$$

The Green-Gauss theorem is applied to this equation, and noting that the initial strain and the boundary integral terms are set to zero, the bilinear form $\mathcal{B}(u, v ; \theta)$ and the linear form $\mathcal{F}(v ; \theta)$ can be defined such that

$$
\begin{aligned}
\mathcal{B}(u, v ; \theta) \stackrel{\text { def }}{=} \int_{\mathcal{D}} \varepsilon(v): a(\mathbf{r}, \theta): \varepsilon(u) d \mathbf{r}+\int_{\mathcal{D}} \rho(\mathbf{r}, \theta) v \cdot \frac{\partial^{2} u}{\partial t^{2}} d \mathbf{r} \\
+\int_{\mathcal{D}} v \cdot \mathcal{L}_{\eta(\mathbf{r}, \theta)} \frac{\partial u}{\partial t} d \mathbf{r} \\
\mathcal{L}(v ; \theta) \stackrel{\text { def }}{=} \int_{\mathcal{D}} v \cdot p d \mathbf{r}
\end{aligned}
$$

The FE approximation of the admissible function space ${ }^{o} E(\mathcal{D})$ can be written as $E(\mathcal{D})_{n} \subset E(\mathcal{D})$ such that $u^{n}=\sum_{i}^{n} \psi(\mathbf{r}) u_{i}(t ; \theta), u_{i} \in \mathbb{R}$. Using this discrete form of $u^{n} \in E(\mathcal{D})_{n}$ in Eqs. (11) and (12) obtains

$$
\mathcal{B}\left(u^{n}, v^{n} ; \theta\right)=\mathcal{L}\left(v^{n} ; \theta\right) \quad \forall v^{n} \in \stackrel{o}{E}(\mathcal{D})_{n}
$$

Denoting the nodal components of the displacement field as $\mathbf{u}(t ; \theta)=\left[u_{1}(t ; \theta), \ldots, u_{n}(t ; \theta)\right]^{T} \in \mathbb{R}^{n}$, the FE system of equations from Eq. (13) is

$$
\mathbf{v}^{T}\left(\sum_{i=0}^{2} \mathbf{A}_{i}(\theta) \mathbf{u}_{, i}(t ; \theta)\right)=\mathbf{v}^{T} \mathbf{p}(t), \quad \forall \mathbf{v} \in \mathbb{R}^{n}
$$

where $\mathbf{A}_{i}(\theta)=$ system matrices (stiffness, damping, mass for $i=0,1$, and 2 , respectively), and $\mathbf{u}_{i}(t ; \theta)=i$ th time derivative of the displacement field. The system matrices inherit the randomness of the input stochastic parameters; hence, the stochastic linear system for structural dynamics takes the form

$$
\mathbf{M}(\theta) \ddot{\mathbf{u}}(t ; \theta)+\mathbf{C}(\theta) \dot{\mathbf{u}}(t ; \theta)+\mathbf{K}(\theta) \mathbf{u}(t ; \theta)=\mathbf{p}(t)
$$

where $\mathbf{M}(\theta), \mathbf{C}(\theta)$, and $\mathbf{K}(\theta)=$ random mass, damping, and stiffness matrices, respectively; $\mathbf{u}(t ; \theta)$ and its dotted variants $=$ system response vector and its time derivatives, respectively; and $\mathbf{p}(t)$ $=$ deterministic forcing vector. Following the discretized spectral representation of the random field in Eq. (7) and the bilinear form in Eq. (11), the system matrices can be expanded in terms of the functions of the input random variables as 


$$
\begin{aligned}
& \mathbf{M}(\theta)=\mathbf{M}_{0}+\sum_{i=1}^{p_{1}} \mu_{i}\left(\theta_{i}\right) \mathbf{M}_{i} \in \mathbb{R}^{n \times n} \text { and } \\
& \mathbf{K}(\theta)=\mathbf{K}_{0}+\sum_{i=1}^{p_{2}} \nu_{i}\left(\theta_{i}\right) \mathbf{K}_{i} \in \mathbb{R}^{n \times n}
\end{aligned}
$$

Here the mass and stiffness matrices have been expressed in terms of their deterministic components $\left(\mathbf{M}_{0}\right.$ and $\left.\mathbf{K}_{0}\right)$ and the corresponding random contributions $\left(\mathbf{M}_{i}\right.$ and $\mathbf{K}_{i}$ ) obtained from discretizing the mass and stiffness parameters with a finite number of random variables $\left[\mu_{i}(\theta)\right.$ and $\left.\nu_{i}(\theta)\right]$. The total number of random variables utilized to represent the stochastic system matrices is $M=p_{1}+p_{2}$. For most cases, the damping parameter is expressed as a linear combination of the mass matrix and the system stiffness matrix, which is the proportional damping model; this has been adopted in the present work.

\section{Solution Technique} sought in the space $L^{2}\left(\Theta ; T \times \mathbb{R}^{n}\right)$, which is the space of real valued square integrable functions defined on the probability space $(\Theta, \mathcal{F}$, $P)$. The nature and characteristics of this function space will be subsequently explored in more detail. A direct time-integration scheme is introduced at first to transform Eq. (15) to a set of linear algebraic equations, which is to be solved at each time step. The solution of this system can then be written to exist in $L^{2}\left(\Theta ; \mathbb{R}^{n} \times t\right)$, where $t \in \mathbb{R}^{+}$denotes the dependence of the FE solution vector on time $t$.

\section{Time-Integration Technique}

The time-domain response of the stochastic linear system in Eq. (15) necessitates a time-integration scheme. This is achieved using different time-stepping techniques, which may be implicit or explicit in nature (based on how the response quantities depend on those obtained at the previous steps). Here, an implicit direct integration operator is used, and the solution methodology for the linear stochastic systems obtained with such an approach is described.
The solution of the stochastic system response $\ddot{\mathbf{u}}(t ; \theta)$ in Eq. (15) is
Next the direct integration operators $\overline{\mathbf{A}}_{1}$ and $\overline{\mathbf{A}}_{0}$ are introduced, which depend on the system matrices, but are independent of the response quantities. The system equation can generally be written in terms of this operator as

$$
\overline{\mathbf{A}}_{1} \overline{\mathbf{u}}\left(t_{s+1}\right)=\overline{\mathbf{p}}\left(t_{s+1}\right)+\overline{\mathbf{A}}_{0} \overline{\mathbf{u}}\left(t_{s}, t_{s-1}, \ldots\right)
$$

where the integration operators transform the system response at the earlier time steps $t_{s}, t_{s-1}, \ldots$ to the repose at $t_{s+1}$. The quantities $\overline{\mathbf{u}}\left(t_{s+1}\right)$ and $\overline{\mathbf{u}}\left(t_{s}, t_{s-1}, \ldots\right)$ are defined as follows:

$$
\begin{aligned}
\overline{\mathbf{u}}\left(t_{s+1}\right)= & \left\langle\mathbf{u}\left(t_{s+1}\right) ; \dot{\mathbf{u}}\left(t_{s+1}\right) ; \ddot{\mathbf{u}}\left(t_{s+1}\right)\right\rangle \\
\overline{\mathbf{u}}\left(t_{s}, t_{s-1}, \ldots\right)=\langle & \left\{\mathbf{u}\left(t_{s}\right) ; \dot{\mathbf{u}}\left(t_{s}\right) ; \ddot{\mathbf{u}}\left(t_{s}\right)\right\} ; \\
& \left.\left\{\mathbf{u}\left(t_{s-1}\right) ; \dot{\mathbf{u}}\left(t_{s-1}\right) ; \ddot{\mathbf{u}}\left(t_{s-1}\right)\right\} ; \ldots\right\rangle
\end{aligned}
$$

For single-step schemes, the response at $t_{s+1}$ depends only on the previous time step $t_{s}$. When the operators $\overline{\mathbf{A}}_{1}$ and $\overline{\mathbf{A}}_{0}$ can be written in upper or lower triangular form (in the block sense), it becomes an explicit time-integration scheme. The stability and convergence behavior of the time-integration scheme will be subsequently discussed.

For the case of stochastic linear systems, the system matrices inherit the parametric randomness; hence, they themselves are random in nature. This leads to the integration operators $\overline{\mathbf{A}}_{1}$ and $\overline{\mathbf{A}}_{0}$ being random in nature. The integration operators are linear combinations of the system matrices and can be expressed as the polynomial series of random variables, as in Eqs. (13) and (14), such that the series expansion takes the form of $\overline{\mathbf{A}}_{1}(\theta)=\mathbb{E}\left[\overline{\mathbf{A}}_{1}(\theta)\right]$ $+\sum_{i} \varphi_{j}\left(\chi_{i}\right)\left(\overline{\mathbf{A}}_{1}\right)_{i}$, where $\left(\overline{\mathbf{A}}_{1}\right)_{i}$ denotes the perturbation components of the integration operator associated with the random variables $\varphi_{j}\left(\chi_{i}\right)$ [Eq. (8)], which inherits the input randomness.

For the present case, the Newmark generalized acceleration operator (Bathe 1996; Hahn 1991) is used, which gives an unconditionally stable time-integration scheme. Other such methods include the Wilson averaging operator and the Houbolt operator (Nickel 1971), both of which offer unconditional stability. The integration operators for the stochastic dynamic case for the Newmark method are of the following form:

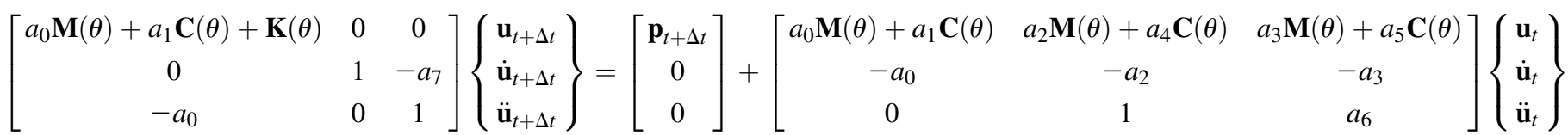

provides unconditional stability under the aforementioned criterion, it is conditionally convergent (Hahn 1991). The convergence is guided by the condition (Newmark 1959)

$$
\frac{\Delta t}{T}<\frac{1}{2 \pi}\left(\frac{1}{\alpha}\right)^{1 / 2} \text { for } \alpha>0
$$

The parameters $\alpha$ and $\gamma$, which have to be chosen, are guided by the consideration of unconditional stability, which is ensured using the following two criterion (Bathe 1996): $\gamma \geq 0.50$ and $\alpha \geq 0.25(0.5+\gamma)^{2}$. Although the implicit Newmark method where $T=$ natural time period of vibration of a single-degree-offreedom (DOF) system. For multiple DOF systems, it is required that 9 $T$ be interpreted as the time period of the highest vibration mode of the system. This results in the choice of time step to be guided by the 10 dimension of the linear system being solved, that is, the higher the 
dimension of the linear system, the lower the value of $T$ (associated with the highest vibration mode) and the smaller the upper bound on the time-step size $\Delta t$. This can have a huge adverse effect for the spectral Galerkin solution technique because the dimension of the linear system increases exponentially with the order of the stochastic polynomials used in the solution basis. Therefore, the PC method would typically require a significantly lower value of the time-step size $\Delta t$ to produce the time-integration results of identical numerical accuracy compared with other techniques, such as direct MCS, which deals with the dimension of the original discretized FE system.

Eq. (19) is rewritten in a more compact form following Eq. (17) as

$$
\overline{\mathbf{A}}_{1}(\theta) \overline{\mathbf{u}}_{t+\Delta t}(\theta)=\overline{\mathbf{p}}_{t+\Delta t}+\overline{\mathbf{A}}_{0}(\theta) \overline{\mathbf{u}}_{t}(\theta)
$$

where $\overline{\mathbf{u}}_{t+\Delta t} \in \mathbb{R}^{N} \times \Theta$ is the ensemble of the stochastic displacement, velocity, and acceleration vector of dimension $N=3 n$ ( $n$ is the dimension of the discretized FE system); the integration operators $\overline{\mathbf{A}}_{1}(\theta) \in \mathbb{R}^{N \times N}$ and $\overline{\mathbf{A}}_{0}(\theta) \in \mathbb{R}^{N \times N}$ are as defined in Eq. (19). This form is used in the subsequent sections to formulate a solution methodology using the different solution methodologies associated with stochastic dynamical systems. Following the Newmark operator given in Eq. (19), the equation for the displacement field in Eq. (15) can be represented to be solved at each time step as

$$
\left[a_{0} \mathbf{M}(\theta)+a_{1} \mathbf{C}(\theta)+\mathbf{K}(\theta)\right] \mathbf{u}_{t+\Delta t}(\theta)=\mathbf{p}_{t+\Delta t}^{\mathrm{eqv}}(\theta)
$$

where $\mathbf{p}_{t+\Delta t}^{\text {eqv }}(\theta)=$ equivalent force at time $t+\Delta t$, which consists of contributions of the system response (displacement, velocity, and acceleration fields) at the previous time step. The equivalent force at each time step becomes a stochastic quantity because of the presence of the stochastic system matrices on the right side and the system response at previous time steps, which themselves are random quantities.

Following the discussion of the expansion of the system matrices in Eq. (15) in terms of their mean and perturbation components, and expressing the structural damping in proportional form, $\mathbf{C}(\theta)$ $=\zeta_{1} \mathbf{M}(\theta)+\zeta_{2} \mathbf{K}(\theta)$, the matrices $\mathbf{A}_{0} \in \mathbb{R}^{n \times n}$ and $\mathbf{A}_{i} \in \mathbb{R}^{n \times n}$; $i=1,2, \ldots, M$ are defined as

$$
\mathbf{A}_{0}=a_{0} \mathbf{M}_{0}+a_{1} \mathbf{C}_{0}+\mathbf{K}_{0}=\left(a_{0}+a_{1} \zeta_{1}\right) \mathbf{M}_{0}+\left(a_{1} \zeta_{2}+1\right) \mathbf{K}_{0}
$$

$$
\mathbf{A}_{i}= \begin{cases}\left(a_{0}+a_{1} \zeta_{1}\right) \mathbf{M}_{i} & \text { for } \quad i=1,2, \ldots, p_{1} \\ \left(1+a_{1} \zeta_{2}\right) \mathbf{K}_{i-p_{1}} & \text { for } \quad i=p_{1}+1, p_{1}+2, \ldots, p_{1}+p_{2}\end{cases}
$$

The linear structural system in Eq. (23) can be expressed as

$$
\underbrace{\left[\mathbf{A}_{0}+\sum_{i=1}^{M} \xi_{i}(\theta) \mathbf{A}_{i}\right]}_{\mathbf{A}(\theta)} \mathbf{u}_{t+\Delta t}(\theta)=\mathbf{p}_{t+\Delta t}^{\text {eqv }}(\boldsymbol{\xi}(\theta))
$$

where $\mathbf{A}_{0}$ and $\mathbf{A}_{i}=$ deterministic and stochastic parts of the system matrices, respectively; $\mathbf{A}_{0}$ and $\mathbf{A}_{i} \in \mathbb{R}^{n \times n} ; i=1,2, \ldots, M=$ symmetric matrices; $\mathbf{u}_{t+\Delta t}(\theta) \in \mathbb{R}^{n}=$ solution vector; and $\mathbf{p}_{t+\Delta t}^{\text {eqv }}(\boldsymbol{\xi}(\theta))$ $\in \mathbb{R}^{n}=$ forcing vector, which comprises of the deterministic forcing function at each time step plus the stochastic system response at earlier time steps. They have a nonlinear functional dependence on the random variables, which have been used to model the parametric uncertainty. The number of terms $M$ in Eq. (25) can be selected based on the accuracy desired for the representation of the underlying random field. This is the specific form of the system matrices under the Newmark time-stepping scheme. However, the general scheme of the solution technique remains the same for this class of implicit timestepping techniques. The expressions for $\mathbf{A}_{0}$ and $\mathbf{A}_{i}$ vary according to the damping model chosen for a particular application. One of the main aims of stochastic dynamic analysis is to obtain $\mathbf{u}_{t+\Delta t}(\theta)$ for $\theta \in \Theta$ and for all time steps $t \in[0, T]$ in an efficient manner, which is the primary motivation for this work.

\section{Overview of the Spectral Galerkin Approach for Structural Dynamics}

Several methods have been utilized to resolve the time-domain response of stochastic systems. These include the direct MCS (Shinozuka 1972), the perturbation-based stochastic FEM method (Wall and Bucher 1987), and the Neumann expansion method (Lei and Qiu 2000). However, the spectral Galerkin methods have been studied with particular enthusiasm over the last two decades. Spectral Galerkin methods are used in conjunction with various timeintegration techniques to evaluate the time-domain response. The long time-integration scheme has been used with gPC in Gerritsma et al. (2010), which shows a growth of error with time. Lucor et al. (2004) implemented the temporal discretization of a single DOF linear oscillator with the implicit Newmark method, which shows that a high polynomial degree is essential for accuracy of the computed results. Xiu and Karniadakis (2003a) considered the generalized chaos for transient response of FE thermal systems with random heat conductivity and capacity. However, the use of implicit time-integration schemes for stochastic FE structural dynamic systems with Galerkin projection schemes remains a sparsely studied area of research. The novelty of the approach described here lies in the formulation of the stochastic time-integration operator in conjunction with the spectral Galerkin approach, and the linear algebraic system that results from the implementation is presented here.

Here the solution of the assembled system of stochastic linear algebraic equations, as given Eq. (22), is presented. From Eq. (25), the appropriate function space in which the solution of the stochastic problem exists can be identified. The random matrices $\mathbf{A}_{i} \in \mathbb{R}^{n \times n}$ inherit the continuity and coercivity properties from the governing SPDE through the weak formulation. The forcing vector $\mathbf{p}_{t+\Delta t}^{\text {eqv }}: \Theta$ $\rightarrow \mathbb{R}^{n}$ depends on the system response at earlier time steps, which in turn can be assumed to be continuous. The spatially discretized solution vector $\mathbf{u}_{t+\Delta t}$ at each time step lies in the tensor product space $\mathbb{R}^{n} \otimes r$, where $r$ is an ad hoc function space for real-valued random variables. Given that the stochastic system has been discretized and represented with a finite number of random variables $\boldsymbol{\xi}(\theta)=\left\{\xi_{1}\right.$, $\left.\ldots, \xi_{M}\right\}$ [as, e.g., in Eq. (25)], the stochastic subspace reduces to $r_{M}$, where $r_{M} \subset r$. When each random component $\xi_{i}$ is independent, then $r_{M}$ is a tensor product space $r^{(1)} \otimes r^{(2)} \otimes \ldots \otimes r^{(M)}$. According to the approximate basis building techniques that focus on expansion of the solution vector using some polynomial functions, the solution vector can be expressed in the form

$$
\mathbf{u}_{t}=\sum_{a \in \mathcal{I}_{M}} \mathcal{H}_{\alpha}(\boldsymbol{\xi}(\theta)) \tilde{\mathbf{u}}_{t, \alpha} ; \quad \tilde{\mathbf{u}}_{t, \alpha} \in \mathbb{R}^{n}
$$

where $\mathcal{H}_{\alpha}(\boldsymbol{\xi}(\theta))=$ basis in $r_{M}, \tilde{\mathbf{u}}_{t, \alpha}=$ set of unknown coefficients to be evaluated, and $\mathcal{I}_{M}=$ subset of $\mathcal{I}$ with cardinal $M$. The form of the polynomial functions $\mathcal{H}_{\alpha}(\boldsymbol{\xi}(\theta))$ used in Eq. (26) varies according to the chosen solution approach, and the well-known spectral approaches, such as PC and gPC, use orthogonal polynomial basis from the Wiener-Askey scheme. When $\boldsymbol{\xi}(\theta)$ is a vector of independent identically distributed Gaussian random variables, the 
functions $\mathcal{H}_{\alpha}$ are finite-order Hermite polynomials, which are orthonormal with respect to the joint probability density function of the input vector $\boldsymbol{\xi}(\theta)$. The same idea can be extended to non-Gaussian random variables, provided that a more generalized functional basis is used; therefore, the orthonormality with respect to the probability density functions can be retained. Suppose the series in Eq. (26) is truncated after $P$ terms. The value of $P$ depends on the number of basic random variables $M$ and the desired order of the PC expansion. There are $P$ number of unknown vectors of dimension $n$. The successive time derivatives of the displacement field can be expressed as

$$
\frac{\partial^{i} \mathbf{u}_{t}}{\partial t^{i}}=\sum_{a \in \mathcal{I}_{M}} \mathcal{H}_{\alpha}(\boldsymbol{\xi}(\theta)) \frac{\partial^{i} \tilde{\mathbf{u}}_{t, \alpha}}{\partial t^{i}} \quad i=1,2
$$

such that the same stochastic basis is used to formulate the time derivatives of the response at all time steps. The inner product in $r_{M} \times \mathbb{R}^{N}$ [where $N$ is the dimension of the ensemble of the displacement, velocity, and acceleration vector in Eq. (22)] is introduced as

$$
\langle\mathbf{v}, \mathbf{u}\rangle \stackrel{\text { def }}{=} \int_{\Theta} \mathbf{v}^{T}(\boldsymbol{\xi}(\theta)) \mathbf{u}(\boldsymbol{\xi}(\theta)) d P_{\xi}=\mathbb{E}\left[\mathbf{v}^{T}(\boldsymbol{\xi}(\theta)) \mathbf{u}(\boldsymbol{\xi}(\theta))\right]
$$

where $\mathbb{E}[\bullet]=$ expectation operator. The Galerkin formulation of Eq. (22) for the single-step time-integration scheme is

$$
\begin{aligned}
& \left\langle\overline{\mathbf{v}}_{t+\Delta t}^{T}, \overline{\mathbf{A}}_{1} \overline{\mathbf{u}}_{t+\Delta t}\right\rangle=\left\langle\overline{\mathbf{v}}_{t+\Delta t}^{T}, \overline{\mathbf{p}}_{t+\Delta t}\right\rangle+\left\langle\overline{\mathbf{v}}_{t+\Delta t}^{T}, \overline{\mathbf{A}}_{0} \overline{\mathbf{u}}_{t}\right\rangle \\
& \quad \forall \overline{\mathbf{v}}_{t+\Delta t} \in \Upsilon_{M} \times \mathbb{R}^{N}
\end{aligned}
$$

where the Galerkin projection is implemented at each time step $t+\Delta t$. The vector $\overline{\mathbf{u}}_{t}$ is as defined in Eq. (18), which is of the form $\overline{\mathbf{u}}_{t}=\left\langle\mathbf{u}_{t} ; \dot{\mathbf{u}}_{t} ; \ddot{\mathbf{u}}_{t}\right\rangle$.

After this, the mean-square error minimization is applied and the unknown vectors are solved from the resulting linear algebraic system. The linear system is given as

$$
\begin{aligned}
& {\left[\begin{array}{cccc}
\overline{\mathbf{A}}_{1_{(0,0)}} & \overline{\mathbf{A}}_{1_{(0,1)}} & \cdots & \overline{\mathbf{A}}_{1_{(0, P)}} \\
\overline{\mathbf{A}}_{1_{(1,0)}} & \overline{\mathbf{A}}_{1_{(1,1)}} & & \overline{\mathbf{A}}_{1_{(1, P)}} \\
\vdots & & \ddots & \vdots \\
\overline{\mathbf{A}}_{1_{(P, 0)}} & \overline{\mathbf{A}}_{1_{(P, 1)}} & \cdots & \overline{\mathbf{A}}_{1_{(P, P)}}
\end{array}\right]\left[\begin{array}{c}
\overline{\mathbf{u}}_{t+\Delta t,(1)} \\
\overline{\mathbf{u}}_{t+\Delta t,(2)} \\
\vdots \\
\overline{\mathbf{u}}_{t+\Delta t,(P)}
\end{array}\right]} \\
& =\left[\begin{array}{c}
\overline{\mathbf{p}}_{t+\Delta t,(1)} \\
\overline{\mathbf{p}}_{t+\Delta t,(2)} \\
\vdots \\
\overline{\mathbf{p}}_{t+\Delta t,(P)}
\end{array}\right]+\left[\begin{array}{cccc}
\overline{\mathbf{A}}_{0_{(0,0)}} & \overline{\mathbf{A}}_{0_{(0,1)}} & \cdots & \overline{\mathbf{A}}_{0_{(0, P)}} \\
\overline{\mathbf{A}}_{0_{(1,0)}} & \overline{\mathbf{A}}_{0_{(1,1)}} & & \overline{\mathbf{A}}_{0_{(1, P)}} \\
\vdots & & \ddots & \vdots \\
\overline{\mathbf{A}}_{0_{(P, 0)}} & \overline{\mathbf{A}}_{0_{(P, 1)}} & \cdots & \overline{\mathbf{A}}_{0_{(P, P)}}
\end{array}\right]\left[\begin{array}{c}
\overline{\mathbf{u}}_{t,(1)} \\
\overline{\mathbf{u}}_{t,(2)} \\
\vdots \\
\overline{\mathbf{u}}_{t,(P)}
\end{array}\right]
\end{aligned}
$$

where $\overline{\mathbf{A}}_{1_{(i, j)}}=N \times N$ blocks coefficient matrix of the linear system of dimension $N P \times N P$, and $\overline{\mathbf{u}}_{t+\Delta t,(i)}$ and $\overline{\mathbf{u}}_{t,(i)}=i$ th components of the system response at time $t+\Delta t$ and $t$, respectively. The coefficient matrices of the linear system in Eq. (30) are block sparse matrices and symmetric in nature. This structure is conducive to the numerical Krylov-based iterative techniques, such as the biconjugate gradient stabilized (BiCGStab) algorithm, which has been used in this work to solve the linear systems resulting from the spectral Galerkin method. The Krylov iterative techniques can exploit the multicore architecture of modern day computational platforms for performing the large matrix-vector operations. The performance improvement obtained with this technique is subsequently illustrated while comparing the computation cost of the different methods.

However, $P$ increases exponentially with the order of chaos and the number of input random variables $M$; therefore, the dimension $N P$ of the linear system obtained from Eq. (29) becomes very high. As a result, several methods have been developed (Sachdeva et al. 2006; Blatman and Sudret 2010) to reduce the computational cost. In the PC-based solution approach, the only information used to construct the basis is the probability density function of the random variables. In context of the discretized Eq. (25), more information, such as the matrices $\mathbf{A}_{i}, i=0,1,2, \ldots M$, is available. Eq. (26) also shows that the stochastic basis is independent of the time step and lacks any adaptive properties, which may contribute to the building up of errors for long time integration. This is because the nonlinear effect of the input random variables on the stochastic system response is compounded with each time step. It may be possible to construct an alternative stochastic basis using the invariant properties of the linear system and the time step. Such an approach is investigated here, where the solution is projected on to a reduced number of eigenbasis obtained from the underlying deterministic system, weighted by a set of highly nonlinear stochastic weighting functions termed as spectral functions.

\section{Spectral Decomposition in Eigenspace: Derivation of the Spectral Functions}

Following the spectral stochastic FE method, an approximation to the solution of Eq. (25) can be expressed as a linear combination of functions of random variables and deterministic vectors. There is a possibility of an optimal spectral decomposition, which has been discussed in Nouy $(2007,2008)$. The aim is to use a small number of terms to reduce the computation without losing the accuracy. Recently, a reduced Galerkin approach for parabolic stochastic systems has been proposed, which uses the eigenvectors of the baseline model as the basis vectors (Adhikari 2011). This approach has been extended to structural dynamic systems in the frequency domain in Kundu and Adhikari (2011). A reference to the idea of stochastic Krylov space is relevant in this context and can help the representation of the response vector in a reduced subspace that can alleviate much of the computational burden. The fundamental idea is to find the solution to a nonsingular linear system of algebraic equation $\mathbf{A x}=\mathbf{b}$ in a Krylov space whose dimension is the degree of the minimal polynomial of A (Ipsen and Meyer 1998).

\section{Definition 1}

A minimal polynomial $F$ of $\mathbf{A}$ is a unique monic polynomial of minimal degree such that $F(\mathbf{A})=0$. This minimal polynomial of 28 degree $m$ can be constructed with the distinct eigenvalues $\left(\lambda_{j}\right)$ of $\mathbf{A}$ as

$$
F(\mathbf{A})=\prod_{j=1}^{d}\left(\mathbf{A}-\lambda_{j} \mathbf{I}\right)^{m_{j}} \quad \text { and } \quad m \equiv \sum_{j=1}^{d} m_{j}
$$

This idea can be used to construct the inverse of a nonsingular matrix $\mathbf{A}$ in terms of the powers of $\mathbf{A}$ as

$$
\mathbf{A}^{-1}=-\frac{1}{\alpha_{0}} \sum_{j=0}^{m-1} \alpha_{j+1} \mathbf{A}^{j}
$$

where the coefficients $\alpha_{i}$ are evaluated from the minimal polynomial given in Definition 1. This idea can be immediately utilized to recognize that the solution vector $\mathbf{x}$ of the equation $\mathbf{A x}=\mathbf{b}$ lies in the Krylov subspace of order $m$ as 


$$
\mathcal{K}_{m}(\mathbf{A}, \mathbf{b})=\operatorname{span}\left\{\mathbf{b}, \mathbf{A b}, \mathbf{A}^{2} \mathbf{b}, \ldots, \mathbf{A}^{m-1} \mathbf{b}\right\}
$$

Now the Krylov subspace dimension is a key factor that can influence the accuracy of the computed response, and it also plays a key role in the computational efficiency of the solution technique, that is, a low degree of the minimal polynomial would imply a small Krylov solution space. This idea is of central importance to the solution technique presented in this paper.

The stochastic linear set of equations presented in Eq. (25), for which a solution of the response vector $\mathbf{u}_{t+\Delta t}(\theta)$ is sought, follows directly from the aforementioned discussion that the solution at each time step $t+\Delta t$ can be projected on to a finite number of basis spanning a stochastic Krylov space, which can be defined at that time step as

$$
\mathcal{K}_{m}[\underbrace{\left(\mathbf{A}_{0}+\sum_{i=1}^{M} \xi_{i}\left(\theta_{i}\right) \mathbf{A}_{i}\right)}_{\mathbf{A}(\theta)}, \mathbf{p}_{t+\Delta t}^{\mathrm{eqv}}(\boldsymbol{\xi}(\theta))]
$$

where $\boldsymbol{\xi}(\theta)=M$-dimensional random vector $\boldsymbol{\xi}(\theta)=\left\{\xi_{1}(\theta), \xi_{2}(\theta)\right.$, $\left.\ldots, \xi_{M}(\theta)\right\}^{T}$. A choice of a finite number of Krylov basis depends on the eigenspectrum of the coefficient of the system matrix $\mathbf{A}(\theta)$. Because the eigenvalues of the coefficient matrix $\mathbf{A}(\theta)$ are distributed over a long interval on the real axis, the required number of basis functions $(m)$ on which the solution would be projected would become close to the number of DOFs $(n)$ of the system. This increases the computational cost substantially and, therefore, is highly undesirable.

To alleviate this problem, it is suggested (Nair and Keane 2002) to use a preconditioned stochastic Krylov space to arrive at a richer stochastic subspace by using the mean of the coefficient matrix as the preconditioner. It helps in transforming $\mathbf{A}(\theta)$ such that the probability density functions of its eigenvalues show a high degree of overlap. However, as the variability of the random field increases, it is desirable to incorporate some of the randomness of the system matrices into the preconditioner such that the order of the spectral basis functions can be kept low. This motivates the authors to use a different preconditioner for the problem, as is demonstrated in subsequent discussions.

To begin with, the eigenvectors $\phi_{k} \in \mathbb{R}^{n}$ of the generalized eigenvalue problem has been utilized

$$
\mathbf{K}_{0} \phi_{k}=\lambda_{k} \mathbf{M}_{0} \phi_{k} ; \quad k=1,2, \ldots n
$$

Because matrices $\mathbf{K}_{0}$ and $\mathbf{M}_{0}$ are symmetric and generally nonnegative definite, the eigenvectors $\phi_{k}$ for $k=1,2, \ldots n$ form a complete basis. In principle, any complete basis can be used. This choice is selected because of the analytical simplicity, as will be subsequently seen. For notational convenience, the matrices of the eigenvalues and eigenvectors are defined as $\boldsymbol{\lambda}_{0}=\operatorname{diag}\left[\lambda_{1}, \lambda_{2}\right.$, $\left.\ldots, \lambda_{n}\right] \in \mathbb{R}^{n \times n}$ and $\boldsymbol{\Phi}=\left[\phi_{1}, \phi_{2}, \ldots, \phi_{n}\right] \in \mathbb{R}^{n \times n}$. The eigenvalues of structural dynamic systems can be ordered in ascending order so $\lambda_{1}<\lambda_{2}<\ldots<\lambda_{n}$. The orthogonality property of the modal matrix $\boldsymbol{\Phi}$ can be used to write $\boldsymbol{\Phi}^{T} K_{0} \boldsymbol{\Phi}=\boldsymbol{\lambda}_{0}$ and $\boldsymbol{\Phi}^{T} \mathbf{M}_{0} \boldsymbol{\Phi}=\mathbf{I}$. Because the damping matrix is assumed to be proportional, the deterministic coefficient matrix given in Eqs. (24) and (25) is reduced to the diagonal form if the modal coordinate transformation is applied. The following transformations are introduced:

$$
\begin{gathered}
\boldsymbol{\Phi}^{T} \mathbf{A}_{0} \boldsymbol{\Phi}=\boldsymbol{\Lambda}_{0} ; \quad \mathbf{A}_{0}=\boldsymbol{\Phi}^{-T} \boldsymbol{\Lambda}_{0} \boldsymbol{\Phi}^{-1} \quad \text { and } \\
\tilde{\mathbf{A}}_{i}=\boldsymbol{\Phi}^{T} \mathbf{A}_{i} \boldsymbol{\Phi} \in \mathbb{R}^{n \times n} ; \quad i=0,1,2, \ldots, M
\end{gathered}
$$

Each diagonal component of $\boldsymbol{\Lambda}_{0}=\operatorname{diag}\left[\Lambda_{0_{1}}, \Lambda_{0_{2}}, \ldots, \Lambda_{0_{n}}\right] \in \mathbb{R}^{n \times n}$, that is, $\Lambda_{0_{i}}$ can also be expressed as a linear function of the eigenvalue $\lambda_{i}$ obtained in Eq. (35). Suppose the solution of Eq. (25) is given by $\mathbf{u}_{t+\Delta t}(\theta)=\left[\mathbf{A}_{0}+\sum_{i=1}^{M} \xi_{i}(\theta) \mathbf{A}_{i}\right]^{-1} \mathbf{p}_{t+\Delta t}^{\text {eqv }}(\boldsymbol{\xi}(\theta))$. Using the aforementioned discussions and the introduced transformations in Eq. (36), the following is obtained:

$$
\begin{aligned}
\mathbf{u}_{t+\Delta t}(\theta) & =\left[\boldsymbol{\Phi}^{-T} \boldsymbol{\Lambda}_{0} \boldsymbol{\Phi}^{-1}+\sum_{i=1}^{M} \xi_{i}(\theta) \boldsymbol{\Phi}^{-T} \tilde{\mathbf{A}}_{i} \boldsymbol{\Phi}^{-1}\right]^{-1} \mathbf{p}_{t+\Delta t}^{\mathrm{eqv}}(\boldsymbol{\xi}(\theta)) \\
& =\boldsymbol{\Phi} \boldsymbol{\Psi}(\boldsymbol{\xi}(\theta)) \boldsymbol{\Phi}^{T} \mathbf{p}_{t+\Delta t}^{\mathrm{eqv}}(\boldsymbol{\xi}(\theta))
\end{aligned}
$$

where $\boldsymbol{\Psi}(\boldsymbol{\xi}(\theta))=\left[\boldsymbol{\Lambda}_{0}+\sum_{i=1}^{M} \xi_{i}(\theta) \tilde{\mathbf{A}}_{i}\right]^{-1}$. Separating the diagonal and off-diagonal terms of the $\tilde{\mathbf{A}}_{i}$ matrices as $\tilde{\mathbf{A}}_{i}=\boldsymbol{\Lambda}_{i}+\boldsymbol{\Delta}_{i}$, $i=1,2, \ldots, M$, where the diagonal matrix is given as $\boldsymbol{\Lambda}_{i}=\operatorname{diag}\left[\tilde{\mathbf{A}}_{i}\right]$ $=\operatorname{diag}\left[\Lambda_{i_{1}}, \Lambda_{i_{2}}, \ldots, \Lambda_{i_{n}}\right] \in \mathbb{R}^{n \times n}$ and the matrix containing only the off-diagonal elements $\boldsymbol{\Delta}_{i}=\tilde{\mathbf{A}}_{i}-\boldsymbol{\Lambda}_{i}$ is such that trace $\left(\boldsymbol{\Delta}_{i}\right)=0$. Using these, it can be written that

$$
\boldsymbol{\Psi}(\boldsymbol{\xi}(\theta))=[\underbrace{\boldsymbol{\Lambda}_{0}+\sum_{i=1}^{M} \xi_{i}(\theta) \boldsymbol{\Lambda}_{i}}_{\boldsymbol{\Lambda}(\boldsymbol{\xi}(\theta))}+\underbrace{\sum_{i=1}^{M} \xi_{i}(\theta) \boldsymbol{\Delta}_{i}}_{\boldsymbol{\Delta}(\boldsymbol{\xi}(\theta))}]^{-1}
$$

where $\boldsymbol{\Lambda}(\boldsymbol{\xi}(\theta)) \in \mathbb{R}^{n \times n}=$ diagonal matrix and $\left.\boldsymbol{\Lambda}(\boldsymbol{\xi}(\theta)) \in \mathbb{R}^{n \times n}\right)$ $=$ off-diagonal only matrix.

The diagonal matrix $\boldsymbol{\Lambda}(\boldsymbol{\xi}(\theta))$ is treated as the preconditioner to the stochastic Krylov space, such that the solution can be projected onto a very few basis functions; however, it would be possible to predict an accurate solution of the response vector using this left preconditioned stochastic Krylov subspace. The diagonal dominance of the matrices $\tilde{\mathbf{A}}_{i}$ is conducive to the approach being proposed here. When the mean of the coefficient matrix has been used as the preconditioner, the terms of the Neumann series also span the left preconditioned stochastic Krylov space (Nair 2002). It can be readily extended to include the case using $\boldsymbol{\Lambda}(\boldsymbol{\xi}(\theta))$ as the preconditioner giving

$$
\begin{aligned}
\mathcal{K}_{m}( & \left.\boldsymbol{\Lambda}^{-1} \boldsymbol{\Psi}, \boldsymbol{\Lambda}^{-1} \mathbf{p}_{t+\Delta t}^{\mathrm{eqv}}\right) \\
= & \operatorname{span}\left\{\boldsymbol{\Phi}^{T} \boldsymbol{\Lambda}^{-1} \boldsymbol{\Phi} \mathbf{p}_{t+\Delta t}^{\mathrm{eqv}}, \boldsymbol{\Phi}^{T} \mathbf{R}(\boldsymbol{\xi}(\theta)) \boldsymbol{\Lambda}^{-1} \boldsymbol{\Phi} \mathbf{p}_{t+\Delta t}^{\mathrm{eqv}},\right. \\
& \left.\boldsymbol{\Phi}^{T} \mathbf{R}(\boldsymbol{\xi}(\theta))^{2} \boldsymbol{\Lambda}^{-1} \boldsymbol{\Phi} \mathbf{p}_{t+\Delta t}^{\mathrm{eqv}}, \ldots, \boldsymbol{\Phi}^{T} \mathbf{R}(\boldsymbol{\xi}(\theta))^{m-1} \boldsymbol{\Lambda}^{-1} \boldsymbol{\Phi} \mathbf{p}_{t+\Delta t}^{\mathrm{eqv}}\right\}
\end{aligned}
$$

where

$$
\mathbf{R}(\boldsymbol{\xi}(\theta))=\left(\boldsymbol{\Lambda}^{-1}(\boldsymbol{\xi}(\theta)) \boldsymbol{\Delta}(\boldsymbol{\xi}(\theta))\right)
$$

Eq. (39) shows that the Krylov basis is the temporally adaptive stochastic basis function because of the equivalent forcing term $\mathbf{p}_{t+\Delta t}^{\text {eqv }}$, which inherits the response characteristics from the previous time step. The equivalent infinite Neumann series representation of this equation is

$$
\boldsymbol{\Psi}(\boldsymbol{\xi}(\theta))=\sum_{s=0}^{\infty}(-1)^{s}[\mathbf{R}(\boldsymbol{\xi}(\theta))]^{s} \boldsymbol{\Lambda}^{-1}(\boldsymbol{\xi}(\theta))
$$

Taking an arbitrary $r$ th element of $\mathbf{u}(t, \theta)$, Eq. (37) can be rearranged to have 


$$
u_{t+\Delta t}^{r}(\theta)=\sum_{k=1}^{n} \Phi_{r k}\left(\sum_{j=1}^{n} \Psi_{k j}(\boldsymbol{\xi}(\theta))\left(\phi_{j}^{T} \mathbf{p}_{t+\Delta t}^{\text {eqv }}(\boldsymbol{\xi}(\theta))\right)\right)
$$

Vector $\boldsymbol{\Gamma}_{t+\Delta t}(\boldsymbol{\xi}(\theta))$ of dimension $n \times 1$ is defined as

$$
\boldsymbol{\Gamma}_{t+\Delta t}(\boldsymbol{\xi}(\theta))=\boldsymbol{\Psi}(\boldsymbol{\xi}(\theta))\left(\boldsymbol{\Phi}^{T} \mathbf{p}_{t+\Delta t}^{\text {eqv }}(\boldsymbol{\xi}(\theta))\right)
$$

where $\boldsymbol{\Gamma}_{t+\Delta t}(\boldsymbol{\xi}(\theta))=$ vector of highly nonlinear functions of the random variables $\left\{\xi_{i}(\theta): i=1, \ldots, M\right\}$ and is expressed in terms of the spectral properties of the system matrices. Combining Eqs. (42) and (43) gives

$$
\mathbf{u}_{t+\Delta t}(\theta)=\sum_{k=1}^{n} \Gamma_{t+\Delta t}^{k}(\boldsymbol{\xi}(\theta)) \phi_{k}
$$

where $\Gamma_{t+\Delta t}^{k}(\boldsymbol{\xi}(\theta))=k$ th element of the vector of stochastic functions contained in $\boldsymbol{\Gamma}_{t+\Delta t}(\boldsymbol{\xi}(\theta))$, and $\phi_{k}=k$ th eigenmode of the deterministic structural system. The time-domain response of the structural system is projected on to the deterministic eigenmodes of the structural system and weighted by a set of spectral functions $\Gamma_{t+\Delta t}^{k}(\boldsymbol{\xi}(\theta))$.

Assume that the series in Eq. (41) is truncated after the $m$ th term. This is equivalent to taking $m$ terms of the minimal polynomial of the left preconditioned stochastic Krylov space. The truncated function is defined as $\boldsymbol{\Psi}^{(m)}(\boldsymbol{\xi}(\theta))=\sum_{s=0}^{m}(-1)^{s}\left[\boldsymbol{\Lambda}^{-1}(\boldsymbol{\xi}(\theta)) \boldsymbol{\Delta}(\boldsymbol{\xi}(\theta))\right]^{s} \boldsymbol{\Lambda}^{-1}$ $(\boldsymbol{\xi}(\theta))$. From this, one can obtain a sequence for different values of $m$ as

$$
\mathbf{u}_{t+\Delta t}^{(m)}(\theta)=\sum_{k=1}^{n} \Gamma_{t+\Delta t}^{k,(m)}(\boldsymbol{\xi}(\theta)) \phi_{k} ; \quad m=1,2,3, \ldots
$$

Because $\theta \in \Theta$ is arbitrary, in comparing Eq. (25) and (37), it is observed that $\mathbf{u}_{t+\Delta t}^{(m)}(\theta)$ is the solution of Eq. (25) for every $\theta$ as $m \rightarrow \infty$. The proposed solution technique is not limited to any particular choice of the probability measure of the input parametric randomness; hence, it is applicable to almost all random fields, as long as the solution exists.

The matrix power series in Eq. (41) is different from the classical Neumann series (Yamazaki et al. 1988) or the spectral Galerkin approach. In the former case, the elements of the matrix power series are polynomials in $\xi_{i}(\theta)$, whereas for the latter case, the solution is projected on to a set of orthogonal polynomials of the input random variables spanning the reduced stochastic solution space. In contrast, the series in Eq. (41) is in terms of $\left[\boldsymbol{\Lambda}^{-1}(\boldsymbol{\xi}(\theta))\right][\boldsymbol{\Delta}(\boldsymbol{\xi}(\theta))]$, where both terms are random and the elements of this matrix series are not simple polynomials in $\xi_{i}(\theta)$, but are in terms of a ratio of polynomials, as seen in the following equations. The convergence of this series depends of the spectral radius of $\mathbf{R}(\boldsymbol{\xi}(\theta))$ in Eq. (40). $\boldsymbol{\Lambda}(\boldsymbol{\xi}(\theta))$ is a diagonal matrix, and its inverse is also a diagonal matrix. Recall that the diagonal of $\boldsymbol{\Delta}(\boldsymbol{\xi}(\theta))$ contains only zeros. A generic term of this matrix can be obtained as

$$
\begin{aligned}
R_{r s}=\frac{\Delta_{r s}\left(1-\delta_{r s}\right)}{\Lambda_{r r}}= & \frac{\sum_{i=1}^{M} \xi_{i} \Delta_{i_{r s}}\left(1-\delta_{r s}\right)}{\Lambda_{0_{r}}+\sum_{i=1}^{M} \xi_{i} \Lambda_{i_{r}}}=\frac{\sum_{i=1}^{M} \xi_{i} \tilde{A}_{i_{r s}}\left(1-\delta_{r s}\right)}{\Lambda_{0_{r}}+\sum_{i=1}^{M} \xi_{i} \tilde{A}_{i_{r r}}} \\
& =\frac{\sum_{i=1}^{M} \xi_{i} \tilde{A}_{i_{r s}}\left(1-\delta_{r s}\right)}{\left(a_{0}+a_{1} \zeta_{1}\right) \lambda_{r}+\left(a_{1} \zeta_{2}+1\right)+\sum_{i=1}^{M} \xi_{i} \tilde{A}_{i_{r r}}}
\end{aligned}
$$

where $\lambda_{r}=r$ th eigenvalue of the deterministic system, as per Eq. (35), and $\delta_{r s}=$ Kronecker delta. In Eq. (46), the spectral radius of $\mathbf{R}$ is also controlled by the diagonal dominance of the $\tilde{\mathbf{A}}_{i}$ matrices. If the diagonal terms are relatively larger than the off-diagonal terms, the series will converge faster, even if the relative magnitude of $\Lambda_{0_{r}}$ is not large. This is true at all time steps.

Eq. (46) also shows that the spectral radius depends on the implicit time-integration parameters $(\alpha$ and $\gamma)$ and the time-step size, $\Delta t$. When Eq. (46) is expressed explicitly in terms of these parameters, the following simplifications occur:

$$
R_{r s}=\frac{\alpha \Delta t^{2}\left(\sum_{i=1}^{M} \xi_{i} \tilde{A}_{i_{r s}}\left(1-\delta_{r s}\right)\right)}{\lambda_{r}+\left(\lambda_{r} \zeta_{1}+\gamma \zeta_{2}\right) \Delta t+\left(\sum_{i=1}^{M} \xi_{i} \tilde{A}_{i_{r r}}\right) \alpha \Delta t^{2}}
$$

This equation is analyzed for the effect of the time-step size on $\mathbf{R}$ for a given fixed structural system. Because the constants $\alpha$ and $\gamma$ are positive quantities, and assuming that the time-step size chosen is generally quite small

$$
R_{r s}=O\left(\Delta t^{2}\right) \quad \text { for } \quad 0<\Delta t \ll 1
$$

This indicates that a smaller time-step size improves the convergence behavior of the spectral functions following Eq. (50). However, smaller time steps incur higher computational cost.

The novelty of the proposed approach for the resolution of the dynamic response of structural systems under the action of transient excitation can be listed as follows:

- The eigenmodes utilized in this problem are the physical vibration modes of the structural system and can be determined experimentally. This offers a significant advantage because of the fact that the spectral functions can now be calculated from experimental measurements and compared with the theoretical values.

- A left preconditioned Krylov subspace projection approach is utilized here with a stochastic preconditioner to express the solution in terms of the highly nonlinear rational functions of the basic random variables.

- The forcing function at each time step inherits the system randomness from the stochastic response of the previous time step; therefore, the stochastic Krylov bases are temporally adaptive.

- The stochastic preconditioner utilized here depends on the timestep size and the integration constants chosen for the problem [as can be seen from Eqs. (22)-(25)], which results in the convergence being a function of the integration parameters.

- The expression for the spectral functions has been expressed explicitly in terms of these parameters and is presented in Eq. (46).

The functions $\Gamma_{t+\Delta t}^{k}(\boldsymbol{\xi}(\theta)), k=1,2, \ldots n$ are called the spectral functions because they are expressed in terms of the spectral properties of the coefficient matrix of the discretized system equation.

In truncating the series in Eq. (41) up to different terms, spectral functions of different orders are defined. Using the expression in Eq. (43), the first-order spectral functions at each time step can be explicitly obtained as

$$
\begin{aligned}
\Gamma_{t+\Delta t}^{k,(1)}(\boldsymbol{\xi}(\theta)) & =\sum_{j=1}^{n} \Psi_{k j}^{(1)}(\boldsymbol{\xi}(\theta))\left(\phi_{j}^{T} \mathbf{p}_{t+\Delta t}^{\mathrm{eqv}}(\boldsymbol{\xi}(\theta))\right) \\
& =\frac{\phi_{k}^{T} \mathbf{p}_{t+\Delta t}^{\mathrm{eqv}}(\boldsymbol{\xi}(\theta))}{\left(a_{0}+a_{1} \zeta_{1}\right) \lambda_{r}+\left(a_{1} \zeta_{2}+1\right)+\sum_{i=1}^{M} \xi_{i}(\theta) \Lambda_{i_{k}}}
\end{aligned}
$$

Here the spectral functions are rational functions of the basic random variables and change at each time step as a result of the associated 
forcing function $\mathbf{p}_{t+\Delta t}^{\text {eqv }}(\boldsymbol{\xi}(\theta))$. The diagonal dominance of the perturbation matrices $\Lambda_{i_{k}}$ ensures that the spectral functions have a high radius of convergence, which can efficiently tackle high degrees of variability of the input randomness. The solution can be expected to be approximated to a high degree of accuracy even with low-order spectral functions.

The vector of spectral functions of order $m$ can be obtained by retaining $m$ terms in the series [Eq. (41)], and can be expressed as

$$
\begin{aligned}
\boldsymbol{\Gamma}_{t+\Delta t}^{(m)}(\boldsymbol{\xi}(\theta))=[ & \mathbf{I}_{n}-\mathbf{R}(\boldsymbol{\xi}(\theta))+\mathbf{R}(\boldsymbol{\xi}(\theta))^{2}-\mathbf{R}(\boldsymbol{\xi}(\theta))^{3} \\
& \left.\ldots m^{\text {th term }}\right] \boldsymbol{\Gamma}_{t+\Delta t}^{(1)}(\boldsymbol{\xi}(\theta))
\end{aligned}
$$

where $\mathbf{I}_{n}=n$-dimensional identity matrix, and $\mathbf{R}$ is defined in Eq. (40). Different terms of this series can be obtained recursively from the previous term (Yamazaki et al. 1988). Because of the availability of the recursive formula, the calculation of the higherorder spectral functions is expected to be less involved. The computational efficiency and accuracy in using different orders of spectral functions to formulate the approximate solution of the stochastic system is subsequently demonstrated with numerical examples. In the following subsection, the calculation of the different orders of the statistical moments of the solution and the closedform expressions utilized to evaluate them is presented.

The computational complexity involved in calculating the proposed spectral function is presented here. If the stochastic FE system solution is projected on to its modal coordinates with the first $n_{r}$ modes [from Eq. (35)], and $N_{s}$ is the number of stochastic samples points where the system is solved in the direct MCS approach, then the total computational complexity is $(T / \Delta t) N_{s} \mathcal{O}\left(n_{r}^{3}\right)$, where $T$ is the total time for which the system response is evaluated, and $\Delta t$ is the time-step size. For the spectral method, the calculation of the various orders of the spectral functions requires the evaluation of the quantity $\boldsymbol{\Lambda}^{-1}(\boldsymbol{\xi}(\theta)) \boldsymbol{\Delta}(\boldsymbol{\xi}(\theta))$ in Eq. (40), whose complexity is given as $\mathcal{O}\left(n_{r}^{2}\right)$. The complete system response resolution using the $m$ th-order spectral functions is $(T / \Delta t) N_{s}(m-1) N_{t} \mathcal{O}\left(n_{r}^{2}\right)$. The computational complexity of the spectral function approach is found to be growing as a cube of the dimension of the reduced eigenspace, which is approximately one order more efficient than the direct MCS calculations.

\section{Statistical Moments of the Response Vector}

Given that the stochastic displacement vector is a function of the sequence of the input random variables, at each time step $t$, the mean and higher-order statistical moments of the solution $\mathbf{u}_{t}^{(m)}: \mathbb{R}^{n} \times \Theta$ $\rightarrow \mathbb{R}^{n}$ about a given point $\boldsymbol{\xi}_{0}$ in the stochastic space can be constructed using the relationship

$$
\Sigma_{p, t}^{(m)}=\int_{\theta}\left(\mathbf{u}_{t}^{(m)}(\boldsymbol{\xi}(\Theta))-\mathbf{u}\left(\boldsymbol{\xi}_{0}\right)\right)^{p} d P(\boldsymbol{\xi}(\theta)) \quad p=1,2,3, \ldots
$$

where $\Sigma_{p, t}^{(m)} \in \mathbb{R}^{n \times n}=p$ th-order statistical moment of the solution about $\mathbf{u}\left(\stackrel{\boldsymbol{\xi}}{0}_{0}\right)=\mathbb{R}^{n}$, calculated with the $m$ th-order spectral function at time step $t$; and $P=$ joint density function of the input random variables. From these expressions, the mean $\left[p=1, \mathbf{u}\left(\xi_{0}\right)=0\right]$ and variance $\left[p=2, \mathbf{u}\left(\boldsymbol{\xi}_{0}\right)=\Sigma_{1, t}^{(m)}\right]$ of the stochastic solution vector can be written as

$$
\Sigma_{1, t}^{(m)}=\sum_{k=1}^{n} \mathbb{E}\left[\Gamma_{t}^{k,(m)}(\boldsymbol{\xi}(\theta))\right] \phi_{k}
$$

$$
\begin{gathered}
\Sigma_{2, t}^{(m)}=\mathbb{E}\left[\left(\sum_{k=1}^{n} \Gamma_{t}^{k,(m)}(\boldsymbol{\xi}(\theta))-\mathbb{E}\left[\Gamma_{t}^{k,(m)}(\boldsymbol{\xi}(\theta))\right]\right) \phi_{k}\right] \\
=\boldsymbol{\Phi} \Xi_{t} \boldsymbol{\Phi}^{T}
\end{gathered}
$$

where $\boldsymbol{\Phi}=$ matrix of eigenvectors; $\Xi_{\mathrm{t}}=$ temporal variancecovariance matrix at time $t$ of the vector $\boldsymbol{\Gamma}_{t}^{(m)}=\left[\Gamma_{t}^{1,(m)}, \Gamma_{t}^{2,(m)}\right.$, $\left.\ldots, \Gamma_{t}^{n,(m)}\right]$, such that $\Xi_{t, i j}=\mathbb{E}\left[\Gamma_{t}^{i,(m)} \Gamma_{t}^{j,(m)}\right]$; and $\mathbb{E}[\bullet]=$ expectation operator associated with the stochastic space. If the components of the random vector $\boldsymbol{\Gamma}_{t}^{(m)}$ are uncorrelated, then $\Xi_{\mathrm{t}}$ becomes a diagonal matrix. However, because the spectral functions considered here are correlated random variables, a fully populated $\Xi_{\mathrm{t}}$ matrix at every time step is obtained. For this correlated nature of the spectral functions, integration in the stochastic space defined in Eq. (51) calls for efficient sampling techniques or metamodeling strategies, which can be used in conjunction with the solution methodology proposed here.

In the following section, some numerical examples are provided to highlight the performance and computational accuracy of the spectral Galerkin approaches and the proposed spectral function approach with the implicit time-integration scheme. The direct MCS results have also been obtained, which serve as the benchmark solution. The appropriate error estimates, convergence behavior, and simulation times of the solution are presented for effective comparison of the different solution methodologies.

Here Algorithm 1 is presented, which summarizes the proposed spectral function approach used to resolve the stochastic system response using an implicit time-integration technique.

\section{Algorithm 1: Transient Stochastic FEM with Spectral Functions}

Input. Choose time-step size $(\Delta t)$ and discretize time axis $T$ into $N_{t}$ points, $N_{t}=T / \Delta t$.

Input. Stochastic input parameter $\boldsymbol{\alpha}(\theta, \mathbf{r}) \in \mathbb{R} \times \Theta^{(M)}$ using KL modes [Eqs. (4)-(7)].

Input. Calculate the system matrices from $\mathcal{B}\left(u^{n}, v^{n} ; \theta\right)=\mathcal{L}\left(v^{n} ; \theta\right)$ using Eqs. (10)-(15).

Input. Choose parameters $\alpha$ and $\beta$ and evaluate integration constants from Eq. (19).

Output. System response $\mathbf{u}_{t+\Delta t}(\theta), \dot{\mathbf{u}}_{t+\Delta t}(\theta), \ddot{\mathbf{u}}_{t+\Delta t}(\theta) \in \mathbb{R}^{n} \times \Theta$ at each $t \in[0, T]$.

1. Evaluate the first $n_{r}$ eigenpairs $\left[\boldsymbol{\lambda}_{0}, \boldsymbol{\Phi}\right]$ from Eq. (35).

2. Construct $\mathbf{R}(\xi(\theta))$ and its successive powers $\mathbf{R}^{k}(\xi(\theta))$ for $k=1, \ldots, m$ using Eq. (40).

3. Initialize $\mathbf{u}_{0}(\theta), \dot{\mathbf{u}}_{0}(\theta), \ddot{\mathbf{u}}_{0}(\theta)$.

4. For $j=1$ to $N_{t}$ do

5. Calculate $\mathbf{p}_{t+\Delta t}^{\text {eqv }}$ with $\mathbf{u}_{t}(\theta), \dot{\mathbf{u}}_{t}(\theta), \ddot{\mathbf{u}}_{t}(\theta)$ following Eqs. (19) and (23).

6. For $r=1$ to $m$ do

7. Construct the Krylov basis following Eq. (39) using $\mathbf{R}^{r}(\xi(\theta))$, $\boldsymbol{\Phi}$, and $\mathbf{p}_{t+\Delta t}^{\text {eqv }}$.

8. Create the $m$ th-order spectral function vector $\boldsymbol{\Gamma}_{t+\Delta t}^{(m)}(\boldsymbol{\xi}(\theta))$ from Eq. (43).

9. End for

10. Project the system response $u_{t+\Delta t}(\theta)$ in the eigenspace using $\boldsymbol{\Gamma}_{t+\Delta t}^{(m)}(\boldsymbol{\xi}(\theta))$ from Eq. (44).

11. Evaluate $\dot{\mathbf{u}}_{t+\Delta t}(\theta), \ddot{\mathbf{u}}_{t+\Delta t}(\theta)$ at $t=j \Delta_{t}$ from Eq. (19).

12. Evaluate the moments of the response using Eqs. (52) and (53).

13. Make $\mathbf{u}_{t}(\theta), \dot{\mathbf{u}}_{t}(\theta), \ddot{\mathbf{u}}_{t}(\theta)$ equal to $\mathbf{u}_{t+\Delta t}(\theta), \dot{\mathbf{u}}_{t+\Delta t}(\theta), \ddot{\mathbf{u}}_{t+\Delta t}(\theta)$, respectively.

14. End for 


\section{Illustrative Example: the Transient Dynamic Analysis of an Euler-Bernoulli Beam}

To demonstrate the applicability of the methods previously detailed, a prototype problem of the dynamic uniplanar flexural vibration of a one-dimensional Euler-Bernoulli cantilever beam is considered. This application is used to demonstrate the effectiveness of the proposed spectral solution method to this class of SPDEs. The solution is obtained for a specified value of the correlation length and for different degrees of variability of the input random field. The spatially varying stochastic field has been discretized using a finite number of zero mean uncorrelated standard Gaussian variables using the KL expansion theorem. For the beam problem, the elastic modulus $E I_{z}$ is chosen as the stochastic parameter. The beam problem has been solved for its transient response in the time domain under the action of an impulse loading. Direct MCS has been performed for these cases and is taken as the benchmark solution with respect to which the appropriateness of the different methods has been analyzed. A comparison between the PC method and the proposed spectral decomposition technique is presented for the beam problem.

The cantilever beam is taken to be clamped at one end (where the displacement and the rotational DOFs are both taken to be zero). Fig. 1(a) shows the configuration of the cantilever beam with an impulse load at its free end in the $z$-direction. The beam bending occurs in the $x-z$ plane. Assume that the bending modulus $\left(E I_{y}\right)$ is a stationary Gaussian random field of the form

$$
E I_{y}(x, \theta)=E I_{0}(1+a(x, \theta))
$$

where $x=$ coordinate along the length of the beam, $E I_{0}=$ mean bending modulus, and $a(x, \theta)=$ zero mean stationary Gaussian random field. Although the Gaussian random field model is not physically meaningful in the strict sense because the physical quantities being modeled are strictly positive, the model has been used extensively in the existing stochastic FE literature (Ghanem and Spanos 1991; Sarkar and Ghanem 2003). However, when the number of KL expansion terms is chosen carefully, the truncated statistical models are strictly positive (Powell and Elman 2008).

The autocovariance function of this random field is assumed to be

$$
C_{a}\left(x_{1}, x_{2}\right)=\sigma_{a}^{2} e^{-\left(\left|x_{1}-x_{2}\right|\right) / \mu_{a}}
$$

where $\mu_{a}=$ correlation length and $\sigma_{a}=\mathrm{SD}$. The following baseline parameters are used: length $L=1 \mathrm{~m}$, cross section $(b \times h)$ is $39 \times 5.93 \mathrm{~mm}^{2}$, and Young's modulus is $E=2 \times 10^{11} \mathrm{~Pa}$. In this study, deflection of the tip of the beam under an impulse load of $\mathbf{I}_{F(t)}=1.0 \mathrm{~N} \cdot \mathrm{s}$ at $t=0$ is considered. In this paper the forcing is 16 assumed to be deterministic in nature. The case when the forcing is random has been treated extensively in the literature within the scope of random vibration (Lin 1967). For example, if the forcing function is independent of the parametric uncertainty, the proposed spectral function approach could be applied at chosen points in the stochastic space associated with the excitation, and the second-order response statistics can be constructed from it. The correlation length considered in the numerical study for comparison with the PC expansion method is $\mu_{a}=L / 2$, and for this case the number of terms retained $(M)$ in the $\mathrm{KL}$ expansion Eq. (7) is two. Therefore, the input stochastic space is two-dimensional in this case. For the FE discretization, the beam is divided into 100 elements. A standard four DOF Euler-Bernoulli beam model is used. After applying the fixed boundary condition at one edge, the number of DOFs of the model is obtained to be $n=200$. It has been verified that this spatial resolution is sufficient to capture the excitation response of the system completely.

The solution obtained with the proposed reduced basis spectral function has been compared with the direct MCS results and the fourth-order PC expansion. The MCS in the stochastic space is performed with 10,000 samples. The calculations have been performed for four values of input SD, $\sigma_{a}=\{0.05,0.10,0.15,0.20\}$, which simulates increasing input uncertainty.

Fig. 1(b) presents the distribution of the natural frequencies of the cantilever beam from the generalized eigenvalue problem given in Eq. (35). The reduced basis of the problem has been chosen based on the time-step size or the sampling frequency of the problem, that is, all the eigenmodes that cover up to $1,200 \mathrm{~Hz}$ have been chosen. The time-step size for the numerical integration scheme has been chosen as $1 / 800 \mathrm{~s}$. A constant modal damping with a $1 \%$ damping factor for all the modes has been applied.

Fig. 2 shows the time-domain response of the deflection of the tip of the cantilever beam under the action of an unit impulse around time $t=0$ for the different input SD values. The proposed spectral method and the direct MCS results shows good agreement at all time steps. However, the solution generated by the PC method, while closely approximating the MCS solution at earlier times, diverges for higher values of $t$. For a high input $\operatorname{SD}\left(\sigma_{a}\right)$, this discrepancy sets in even earlier.

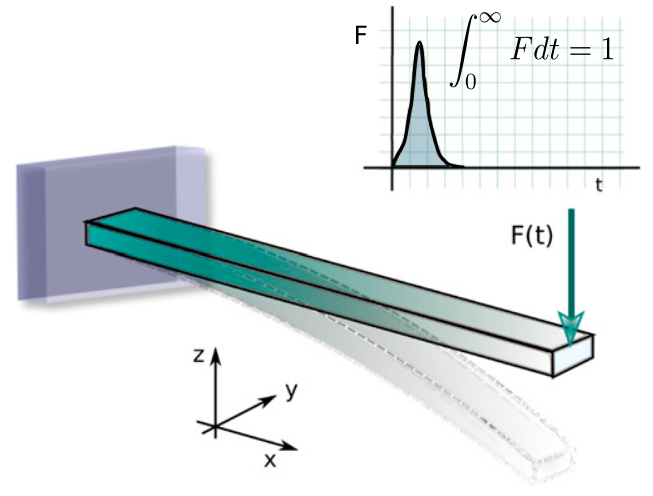

(a)

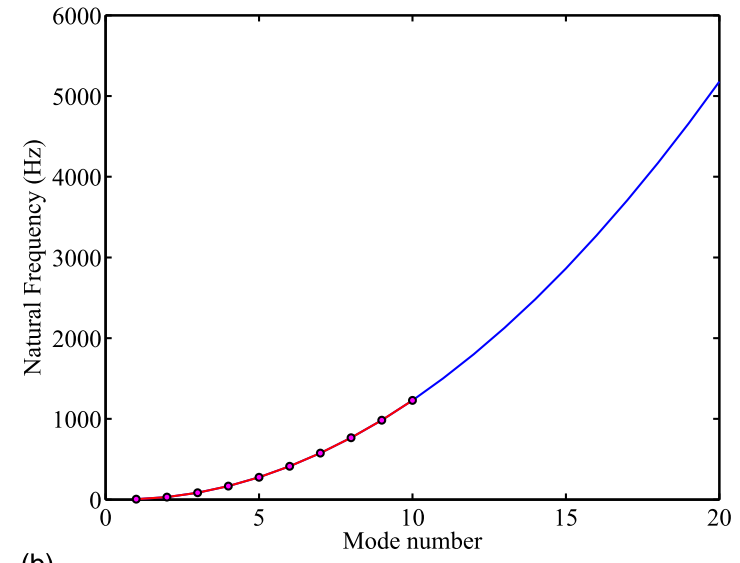

(b)

Fig. 1. Schematic diagram of the cantilever beam with a point load at the free end along with its natural frequencies; the number of reduced eigenvectors chosen is $q=10$, which covers the frequency of up to $1,200 \mathrm{~Hz}$; the fundamental frequency is found to be $4.85 \mathrm{~Hz}$ 
Similar behavior is seen in Fig. 3 in the plot for SD of the deflection of the free end of the beam. The values predicted by the proposed spectral function method are in good agreement with the direct MCS results, whereas the fourth-order PC results show inconsistencies, especially for longer values of $t$. The accurate prediction of higher-order moments using the spectral Galerkin approaches requires a high order of the chaos to be used with the solution. It is expected that the higher moments of the response would tend to deviate significantly.

In Fig. 2, the higher values of input SD produce an effect equivalent to that of damping on the mean deflection values. This is because the randomness in the system parameters tends to distribute the peak response around a neighborhood of the resonance frequency of the deterministic system, and the subsequent averaging smooths out or damps the response at those frequencies. The response is largely comprised of the fundamental frequency $(4.85 \mathrm{~Hz})$ of vibration of the beam because the higher-order modes decay out rapidly. Although the mean response for higher values of $\sigma_{a}$ shows an added damping-kind behavior, in reality any random sample would still produce high levels of vibration in the response.
The fact that the higher-order moments are not properly reproduced by the fourth-order PC method is also verified from the plots of the probability density function of the deflection of the free end of the beam at $t=0.119$ and $0.134 \mathrm{~s}$, as shown in Fig. 4. Although the mean value is closely approximated by the PC solution, the values of the higher-order moments are significantly different from those obtained with the MCS and spectral methods. A very good agreement of the density functions given by the latter two methods is found. The error in the density function produced using the fourthorder PC is much larger (compared with the direct MCS solution) at $t=0.134 \mathrm{~s}$ in Fig. 4(b) than at $t=0.119 \mathrm{~s}$ in Fig. 4(a). This indicates that as the time integration proceeds, the polynomial order of the solution needs to be enhanced to account for the compounded stochastic nonlinearity of the transient system. Similar observations have been reported in the literature (Najm 2009; Gerritsma et al. 2010) for long time integration, which tends to generate unacceptable error levels if higher-order chaos functions are not used for projection of the solution in the stochastic subspace. This, however, has detrimental effects in terms of the computational efficacy of the solution. Hence, it might be desirable to have some
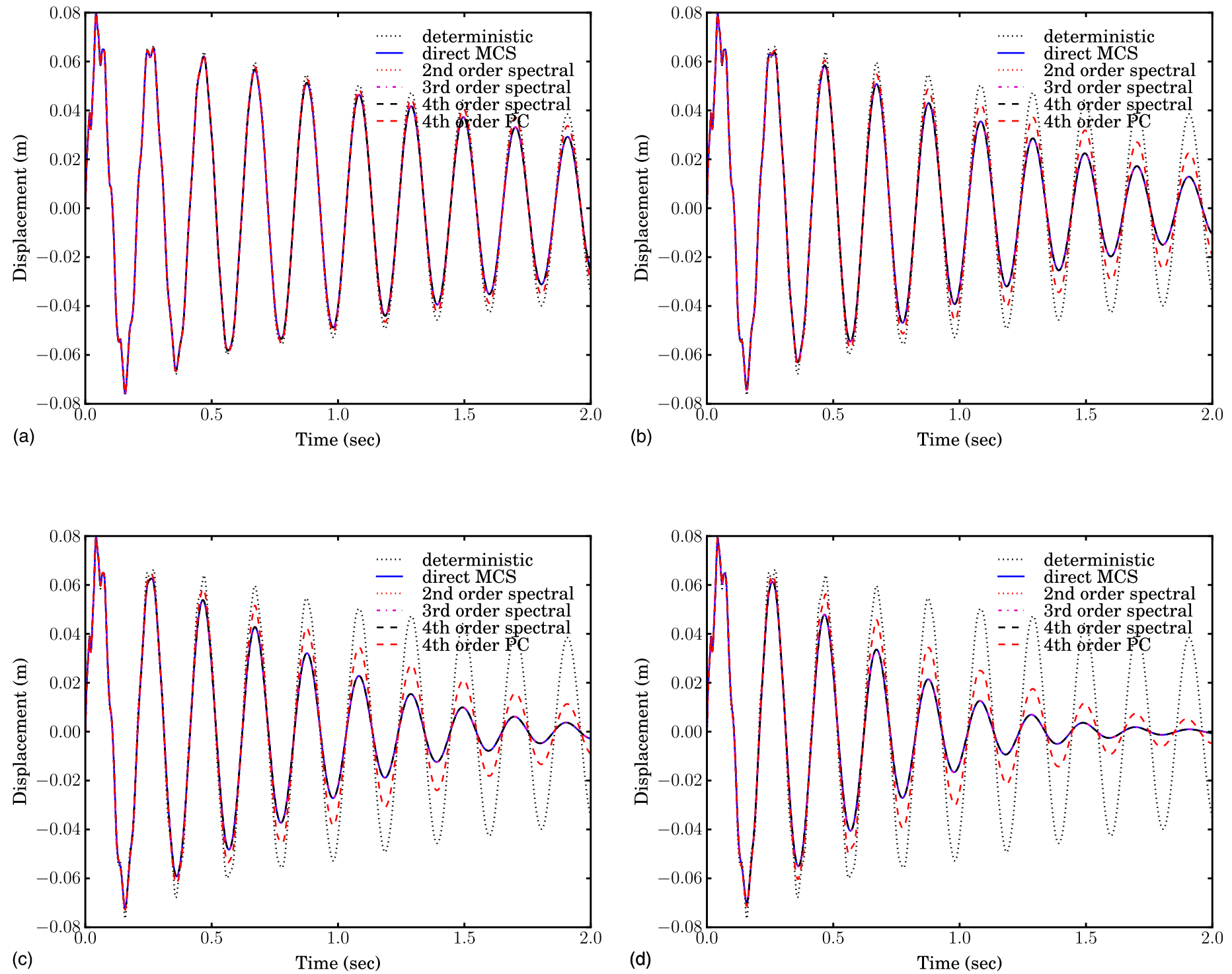

Fig. 2. Mean deflection of the free end of the cantilever beam under an unit impulse load at time $t=0$ for the duration of $1 / 800 \mathrm{~s}$; the response of the reduced-order spectral function method is obtained with 10,000 samples and for $\sigma_{a}=\{0.05,0.10,0.15,0.20\}$ 

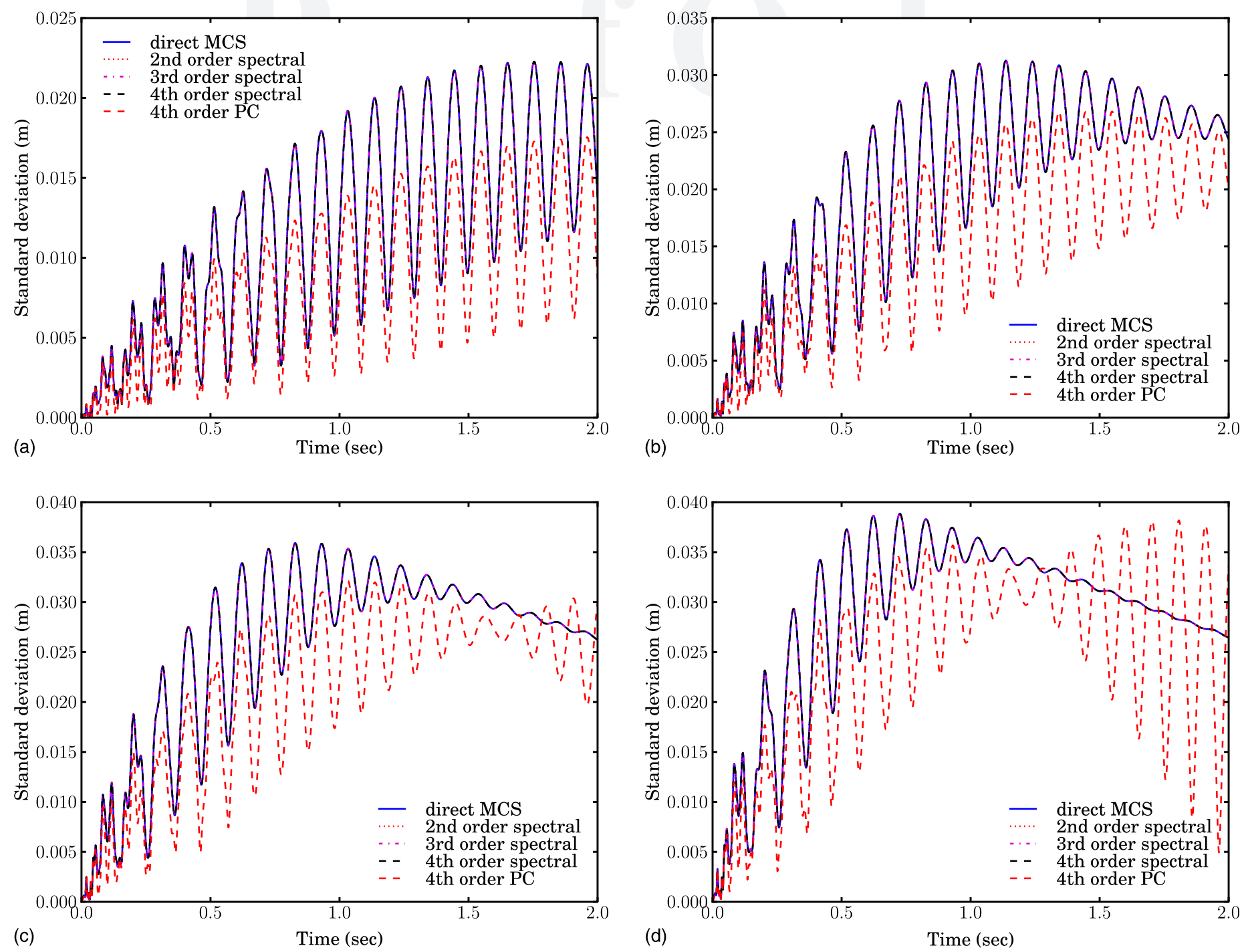

Fig. 3. SD of the deflection of the free end of the cantilever beam under unit impulse load at time $t=0$ for the duration of $1 / 800 \mathrm{~s}$; the response of the reduced-order spectral function method is obtained with 10,000 samples and for $\sigma_{a}=\{0.05,0.10,0.15,0.20\}$

time-adaptivity characteristics in the stochastic basis functions. The accurate estimation of the density curve using a different order of spectral functions is found to be highly conducive to this effect because the stochastic spectral functions change with each time step.

Table 1 presents a comparison of the calculation time of the different methods used in this study to demonstrate the relative computational efficacy of the proposed method. The calculation times are shown for a single time step, performed on a single core of a computational platform. The last entry fourth-order PC (parallel BiCGStab) denotes the time taken in solving the linear block-sparse system obtained from the spectral Galerkin approach using a parallel implementation of the BiCGStab algorithm on eight computational cores, where each core is identical to the single core used in solving the other linear systems mentioned in the table. The table shows that the second-order spectral function approach is, on average, 10 times more efficient than the direct MCS and about 3.5-4 times more efficient than the fourth-order PC method. When the PC is implemented with parallel BiCGStab, the speed obtained is around that of the second-order method. As the order of the spectral function is increased, the computational time increases with it. The performance of the spectral function method can potentially be enhanced significantly using the efficient sampling techniques. The choice of the fourth-order PC to compare the accuracy of the results is justified from this comparison of computational efficacy.

The ACF, which is useful for identifying the relationship between time signals separated by a finite space in time $\tau$, is defined as

$$
\operatorname{ACF}(t, \tau)=\frac{E\left[\left(u_{t}-\mu_{t}\right)\left(u_{t+\tau}-\mu_{t+\tau}\right)\right]}{\sigma_{t} \sigma_{t+\tau}}
$$

where $u_{t}=$ system response at time $t, \sigma_{t}=\mathrm{SD}$ of the response at time 17 $t$, and $\mathbb{E}[\bullet]=$ expectation operator defined over the sample space. The ACF gives important information about the harmonic components contained in the signal and the stationarity of the signals. Fig. 5 shows the autocorrelation surface of the transient response of the free end of the cantilever beam under the unit impulse load for two different values of SD of the input randomness $\left[\sigma_{a}=0.15\right.$ in Fig. 5(a) and $\sigma_{a}=0.20$ in Fig. 5(b)]. The ACF surface is plotted against time $t$ and parameter $\tau$ for the length of the time-domain response $(0.0-2.0 \mathrm{~s})$, as shown in Figs. 2 and 3. The ACF surface is triangular in shape, that is, time $t$ and parameter $\tau$ vary between 0.0 and $2.0 \mathrm{~s}$. The definition of the ACF in Eq. (56) ensures that the ACF response surface remains between -1 and 1 because of normalization with the SD of the response. For very small values of $\tau$, the 


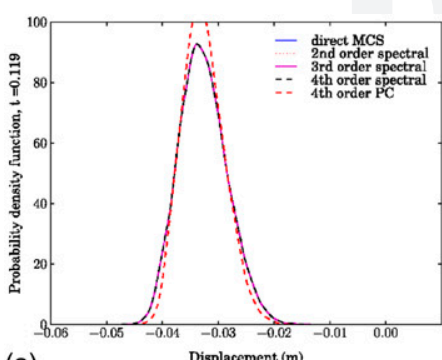

(a)

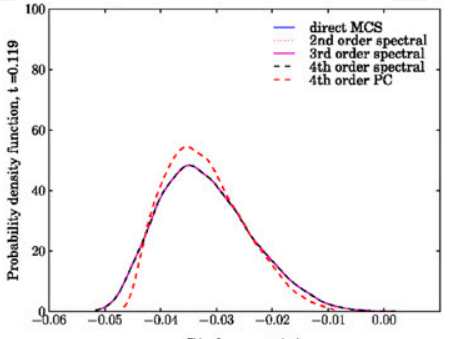

Displacement (m)

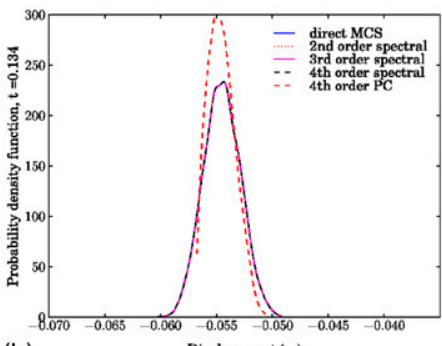

(b)

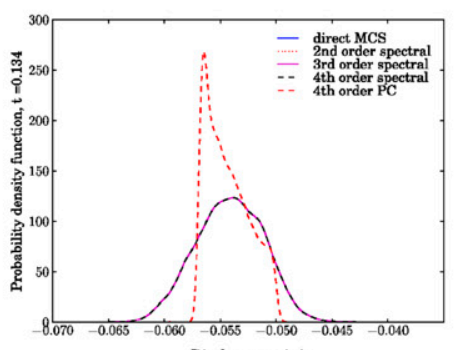

Displacement (m)

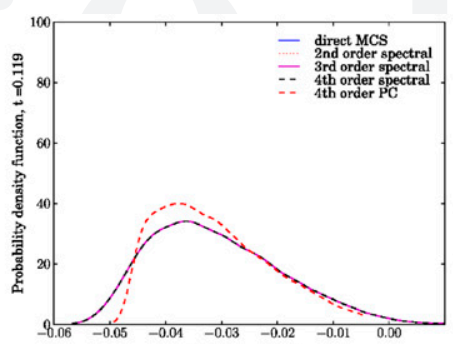

Displacement (m)

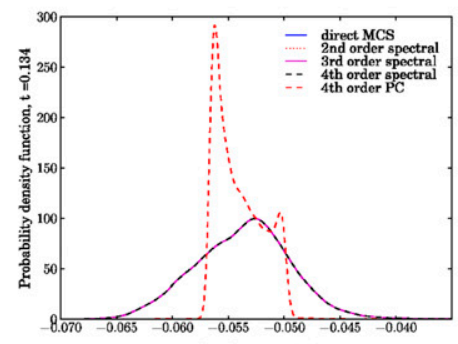

Displacement (m)

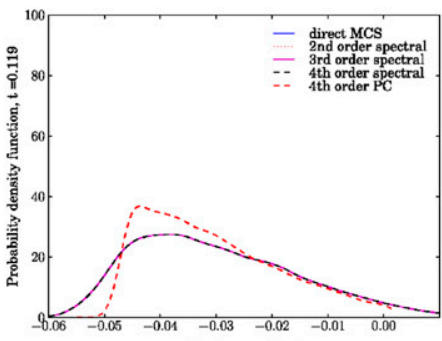

Displacement (m)

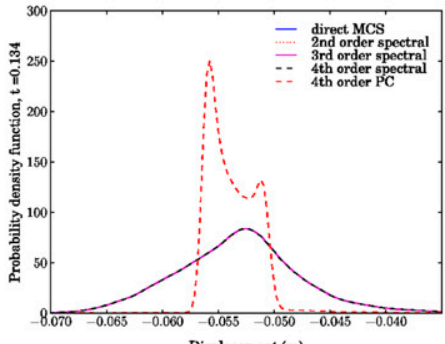

Displacement (m)

Fig. 4. Probability density function of the deflection of the free end of the cantilever beam at $t=0.119$ and $0.134 \mathrm{~s}$ under a unit impulse load at time $t=0$ for a duration of $1 / 800 \mathrm{~s}$; the response of the reduced-order spectral function method is obtained with 10,000 samples and for an input SD of $\sigma_{a}=\{0.05,0.10,0.15,0.20\}$ of the parametric random field

Table 1. Comparison of Calculation Time (in Seconds) of the Proposed Reduced-Order Spectral Function Approach with Direct MCS and FourthOrder PC

\begin{tabular}{lrrr}
\hline Calculation & $\begin{array}{c}\text { Average } \\
\text { time }(\mathrm{s})\end{array}$ & $\begin{array}{c}\text { Minimum } \\
\text { time }(\mathrm{s})\end{array}$ & $\begin{array}{c}\text { Maximum } \\
\text { time }(\mathrm{s})\end{array}$ \\
\hline Direct MCS & 13.589 & 13.506 & 13.798 \\
Second-order spectral & 1.375 & 1.345 & 1.396 \\
Third-order spectral & 1.445 & 1.414 & 1.465 \\
Fourth-order spectral & 1.500 & 1.481 & 1.523 \\
Fourth-order PC & 5.117 & 4.975 & 5.327 \\
Fourth-order PC (parallel BiCGStab) & 1.329 & 1.201 & 1.477 \\
\hline
\end{tabular}

2627 Note: All calculations were performed using a single processor core. The last entry fourth-order PC (parallel BiCGStab) indicates the block sparse linear system solved with parallelized the BiCGStab algorithm on eight computational cores. stationary as the value of $t$ increases, which indicates the transition of the transient response toward a steady state. In other words, the ACF becomes a function of the parameter $\tau$ only for sufficiently large values of $t$. The fourth-order PC, however, does not produce this tendency toward stationarity, which is contrary to the physics of the structural dynamic system. This discrepancy can be attributed to the growing error associated with the time-integration scheme and would have to be addressed with an enhanced order of the PC expansion.

The Galerkin method involved in approximating the solution with the fourth-order Hermite polynomials requires the solution of a linear system of equation of size $3,000 \times 3,000$ at each time step (compared with the original discretized FE system of 200 DOFs). This can impose a stringent condition on the upper bound of the timestep size $\Delta t$ and, as explained in context of Eq. (21), can result in a growth of the error associated with the time-integration scheme. This can lead to a growth of the error associated with spectral Galerkin methods faster than that associated with other samplingbased techniques, where the dimension of the linear system to be solved remains the same as the original deterministic system. Similar behavior is also observed in the SD results shown in Fig. 3, where for higher values of variability of the input randomness (indicated by large $\sigma_{a}$ ), there is a tipping point beyond which the discrepancy of the fourth-order PC result grows, and this point arrives earlier for higher values of input variability. This can be tackled with increasedorder PC expansion, however, the high dimension of the resulting linear system can significantly increase the computational cost of the time-integration scheme because of the enhanced limitation on the maximum time-step size.

The convergence behavior of the proposed spectral function approach with an order of expansion of the spectral functions can be studied with an error indicator. A relative $L^{2}$ error for the system response $\epsilon_{\mathbf{\Sigma}_{j}}^{(m)}(t)$, at each time step $t$ for $m$ th-order spectral function is defined as

$$
\epsilon_{\mathbf{\Sigma}_{j}}^{(m)}(t)=\frac{\left\|\boldsymbol{\Sigma}_{j S F}^{(m)}(t)-\boldsymbol{\Sigma}_{j \operatorname{MCS}}(t)\right\|_{L^{2}(\mathcal{D})}}{\left\|\boldsymbol{\Sigma}_{j \operatorname{MCS}}(t)\right\|_{L^{2}(\mathcal{D})}} \quad \text { for } j=1,2
$$
very good agreement with the direct MCS results. The fourth-order results seen in Fig. 2). Also, from Fig. 6(b), for very small values of $\tau$, the correlation is quite high and the time response becomes almost 

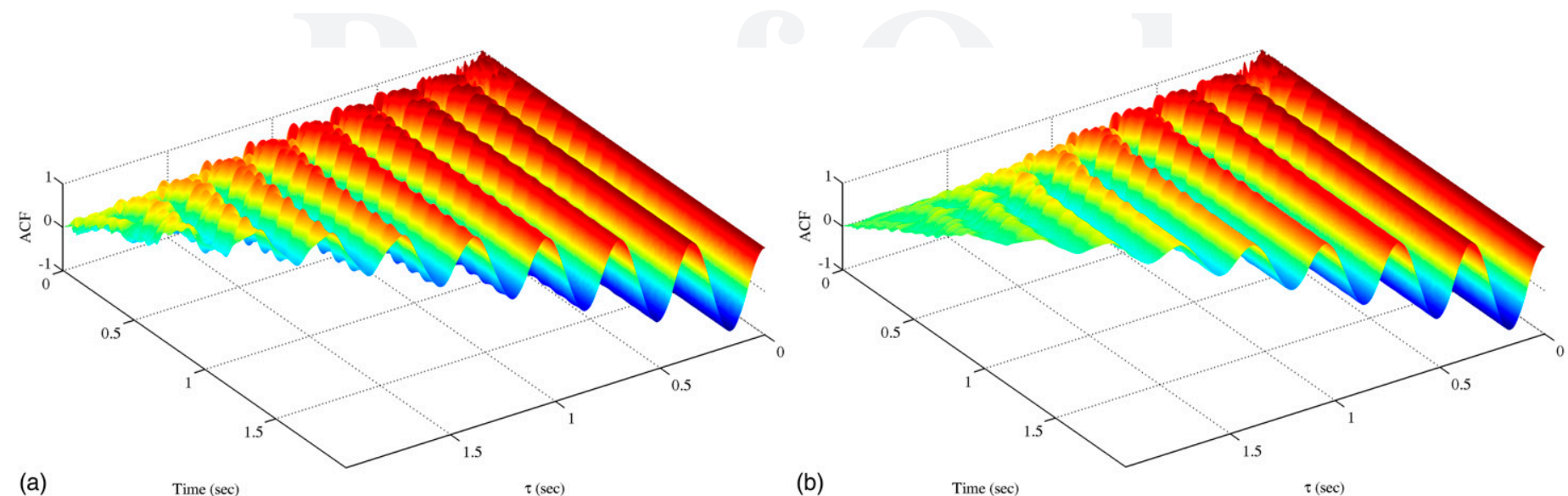

Fig. 5. ACF of the beam response under an unit impulse load at time $t=0$ as a function of $\tau$ and $t$ as given in Eq. (56); the ACF surface has been obtained with direct MCS and is very closely approximated by the results obtained with the spectral function approach
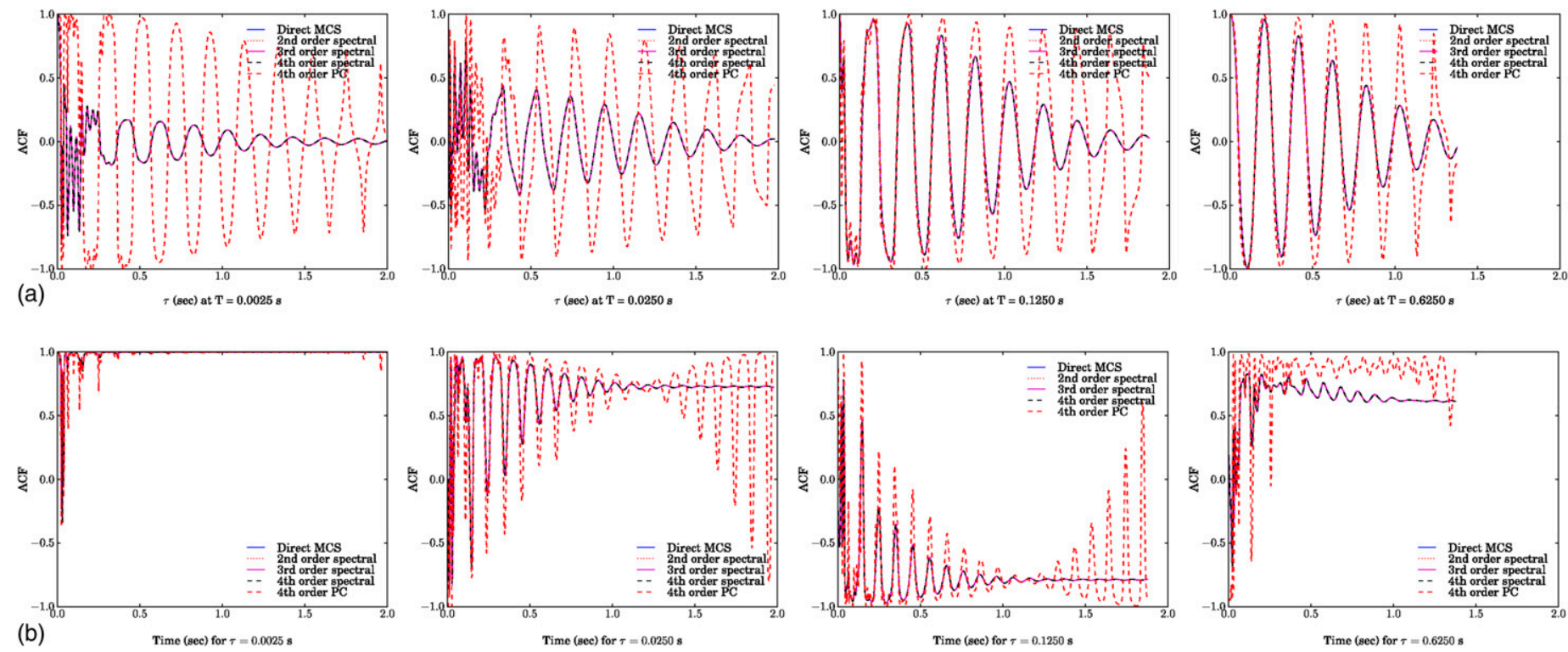

Fig. 6. ACF of the cantilever beam response under an unit impulse load at time $t=0$ for input parametric randomness of $\sigma_{a}=0.20$

where $\boldsymbol{\Sigma}_{j S F}^{(m)}(t)$ is the mean $(j=1)$ or the $\operatorname{SD}(j=2)$ of the system response vector, and $\boldsymbol{\Sigma}_{j \mathrm{MCS}}(t)$ is the same calculated with the direct MCS result. The norm $L^{2}(\mathcal{D})$ covers the discretized FE spatial domain $\mathcal{D}$. Here, the cases for which the spectral function order varies as $m=1, \ldots, 8$ are studied, and the convergence of the $L^{2}$ relative error as functions of the spectral function order are presented.

For the sake of rigor, the authors study a beam vibration problem where a shorter correlation length of the input random parameter (bending modulus) has been assumed. This results in an increase in dimension of the input stochastic space such that for the same approximation error to have a higher value of $m$ is needed while choosing a finite spectrum from the KL expansion in Eq. (7). In the present case, the correlation length is taken to be $\mu_{a}=L / 5$ in Eq. (55); for this, the random bending modulus $E I_{y}$ is approximated with 20 random variables using the KL expansion. Fig. 7 shows the $L^{2}$ error calculated with the mean and SD of the response for different orders of the spectral functions with time $t$ for the highest value of the input randomness considered in this study $\left(\sigma_{a}=0.20\right)$. The higherorder spectral functions definitely provide a better approximation of the results. The same behavior is highlighted at certain time steps, $t=1.375$ and $2.000 \mathrm{~s}$, in Fig. 8 for different degrees of variability of input randomness $\sigma_{a}=\{0.05,0.10,0.15,0.20\}$. However, the higher-order spectral functions have enhanced computational cost associated with them and have to be chosen prudently. The error norm can be used to determine a desired order of expansion for approximating the solution of the system at each time step.

The aforementioned results show that the solution obtained using the spectral functions and a set of orthonormal vector basis functions is well suited for obtaining the unsteady dynamic response of random structural systems, both in terms of accuracy and computational efficiency. Compared with the direct MCS solution method or the spectral Galerkin techniques, the computational cost is favorable because of the reduced order of the system and the finite-order approximation of the solution in the stochastic space. It is found that the estimated solution closely matches the direct MCS solution at all time steps for all values of variability of the system parameters. For the PC method, the growth in the errors on time integration has to be eliminated with enhanced-order expansion of the stochastic basis, which incurs a heavy computational cost. The possibility of using 

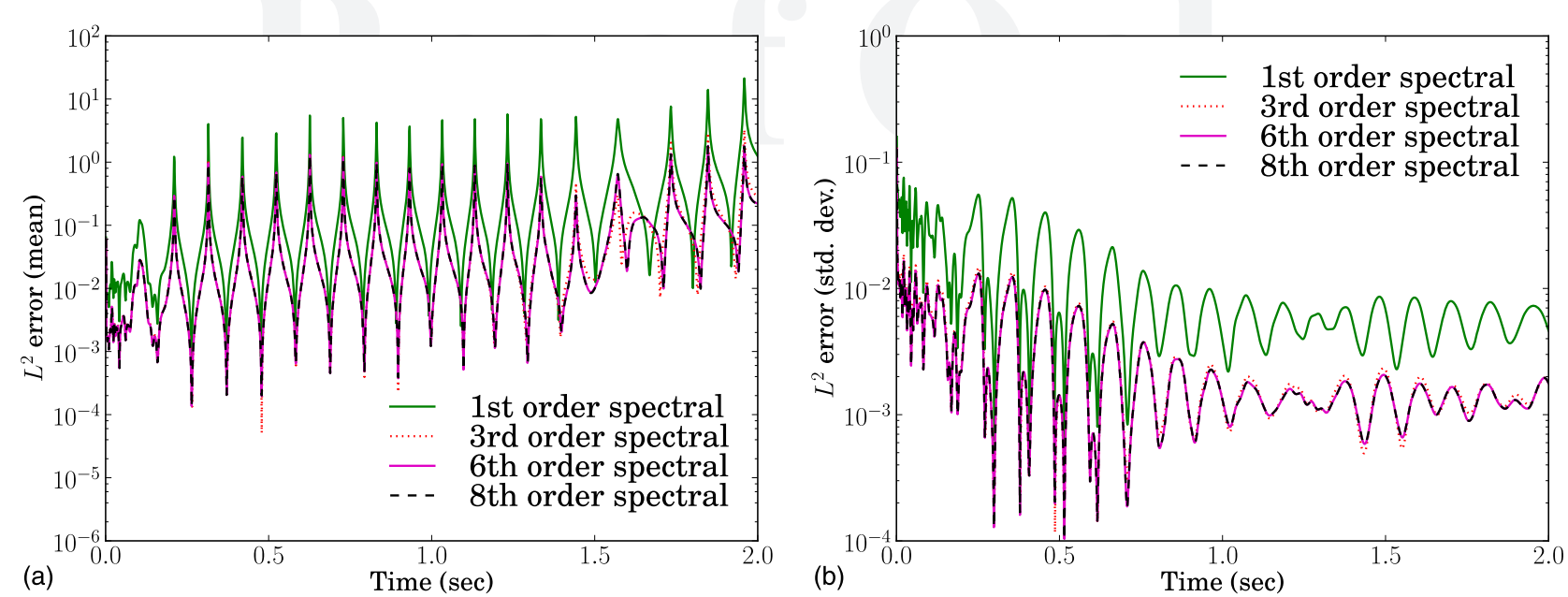

Fig. 7. $L^{2}$ relative error calculated with the mean (a) and SD (b) of the cantilever beam response for different orders of expansion of the spectral functions for an input random field variability of $\sigma_{a}=0.20$
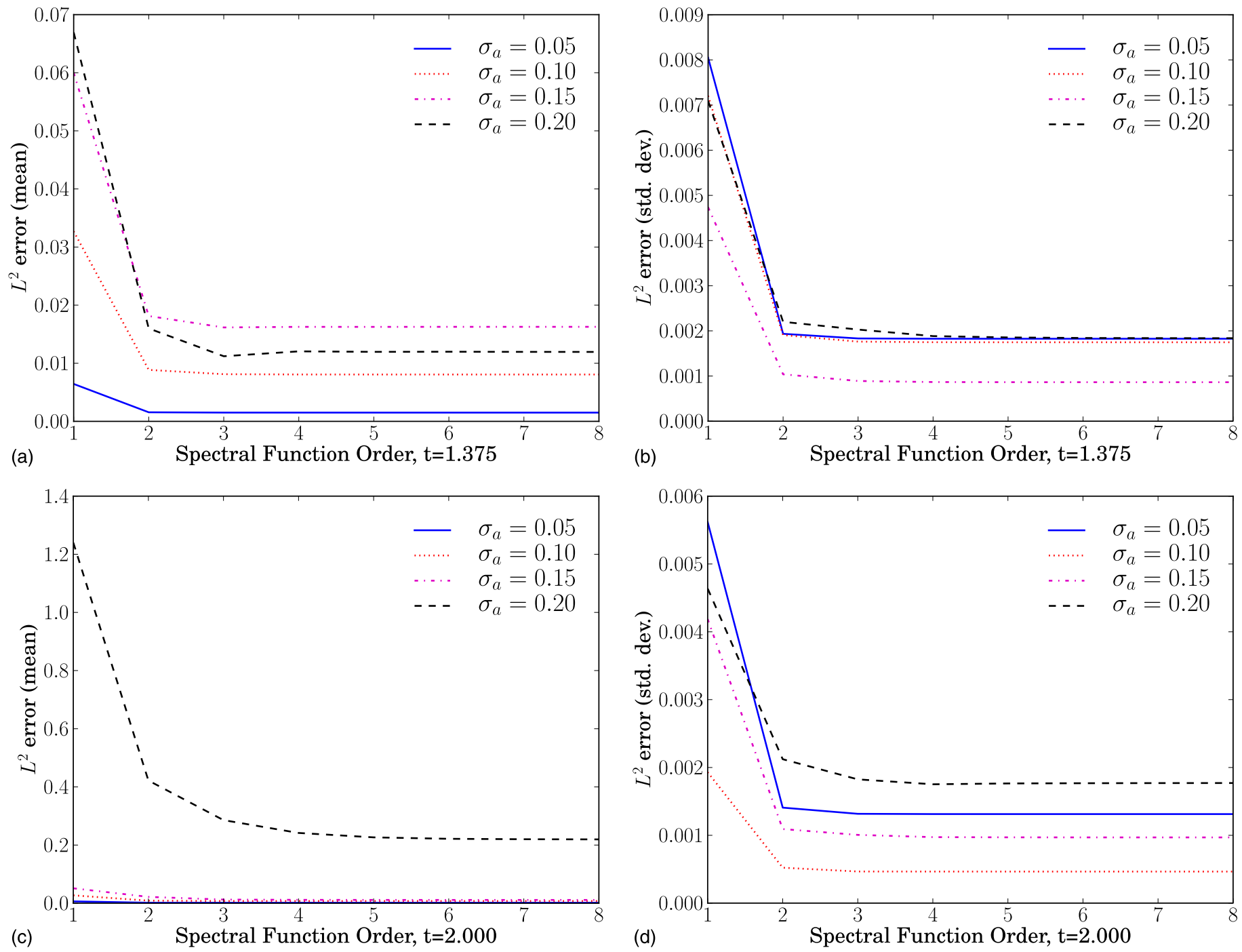

Fig. 8. $L^{2}$ relative error calculated with the mean [(a) and (c)] and SD [(b) and (d)] of the cantilever beam response at $t=1.375$ and $2.000 \mathrm{~s}$ for different orders of expansion of the spectral functions 
the parallel implementations of the iterative linear system solvers to address the issue of computational cost is highly promising.

\section{Conclusions}

Two distinct solution strategies for the resolution of the transient response of stochastic dynamic systems have been proposed. In the classical spectral stochastic finite-element approach, the solution is projected on to a finite set of orthonormal basis functions spanning a reduced stochastic space using a finite order of the chaos functions from the Wiener-Askey scheme. Second, an efficient reduced-order left preconditioned Krylov subspace projection of the stochastic solution on to a finite set of deterministic eigenbasis, which are weighted by dynamic stochastic coefficient functions known as spectral functions, is proposed. A single-step implicit unconditionally stable time-integration scheme has been utilized here with the integration operators being stochastic in nature. Hence, the approach utilizes stochastic temporally adaptive Krylov bases.

The results obtained with the spectral function approach demonstrate good agreement with the direct MCS at all time steps and for different values of input SD $\sigma_{a}$. The fourth-order PC, on the other hand, shows a rapid growth of error for long time integration and for higher-order moments of the solution, which has to be addressed with higher-order chaos expansions, which incur higher computational cost (as found in Table 1). Also, the high dimension of the linear algebraic system encountered in the PC expansion restricts the time-step size $\Delta t$ of the time-integration scheme, which further enhances the associated computational cost. This demonstrates the applicability and computational efficacy of the proposed spectral function approach in context of the unsteady dynamical response of stochastic structural systems.

The future work along this direction would look to improve the proposed stochastic Krylov subspace projection approach using more efficient reduced-order stochastic rational interpolation functions, which could ensure faster convergence of the solution. Further research on a priori error analysis and study of convergence behavior can also give important intuitive guidance in moving toward a choice of a more efficient set of stochastic functions suitable for this class of stochastic problems. Finally, the extension of the proposed methodology to nonlinear structural dynamics problems could constitute an interesting research avenue.

\section{Acknowledgments}

Abhishek Kundu acknowledges the support of Swansea University through the award of a Zienkiewicz Scholarship. Sondipon Adhikakri gratefully acknowledges the support of The Royal Society of London through the Wolfson Research Merit Award.

\section{References}

Adhikari, S. (2011). "Stochastic finite element analysis using a reduced orthonormal vector basis." Comput. Methods Appl. Mech. Eng., 200(21-22), 1804-1821.

Babuska, I., Tempone, R., and Zouraris, G. E. (2005). "Solving elliptic boundary value problems with uncertain coefficients by the finite element method: The stochastic formulation." Comput. Methods Appl. Mech. Eng., 194(12-16), 1251-1294.

Bathe, K. J. (1996). Finite element procedures, Prentice Hall, NJ.

Blatman, G., and Sudret, B. (2010). "An adaptive algorithm to build up sparse polynomial chaos expansions for stochastic finite element analysis." Probab. Eng. Mech., 25(2), 183-197.
Bobrowski, A. (2005). Functional analysis for probability and stochastic processes: An introduction, Cambridge University Press.

Bucher, C., and Bourgund, U. (1990). "A fast and efficient response surface approach for structural reliability problems." Struct. Saf., 7(1), 57-66.

Caflisch, R. E. (1998). "Monte Carlo and quasi-Monte Carlo methods." Acta Numer., 7, 1-49.

Deb, M. K., Babuska, I. M., and Oden, J. T. (2001). "Solution of stochastic partial differential equations using Galerkin finite element techniques." Comput. Methods Appl. Mech. Eng., 190(48), 6359-6372.

Falsone, G., and Impollonia, N. (2002). "A new approach for the stochastic analysis of finite element modelled structures with uncertain parameters." Comput. Methods Appl. Mech. Eng., 191(44), 5067-5085.

Gerritsma, M., van der Steen, J.-B., Vos, P., and Karniadakis, G. (2010). "Time-dependent generalized polynomial chaos." J. Comput. Phys., 229(22), 8333-8363.

Ghanem, R., and Spanos, P. D. (1991). Stochastic finite elements: A spectral approach, Springer-Verlag, New York.

Hahn, G. D. (1991). "A modified Euler method for dynamic analyses." Int. J. Numer. Methods Eng., 32(5), 943-955.

Hurtado, J., and Barbat, A. (1998). "Monte Carlo techniques in computational stochastic mechanics." Arch. Comput. Meth. Eng., 5(1), 3-29.

Ipsen, I. C. F., and Meyer, C. D. (1998). "The idea behind Krylov methods." Am. Math. Mon., 105(10), 889-899.

Kiureghian, A. D., and Ditlevsen, O. (2009). "A leatory or epistemic? Does it matter?" Struct. Saf., 31(2), 105-112.

Kleiber, M., and Hien, T. D. (1992). The stochastic finite element method, Wiley, New York.

Kleijnen, J. (2009). "Kriging metamodeling in simulation: A review." Eur. J. Oper. Res., 192(3), 707-716.

Kundu, A., and Adhikari, S. (2011). "A novel Galerkin projection approach for damped stochastic dynamic systems." ECCOMAS Thematic Conf. on Computational Methods in Structural Dynamics and Earthquake Engineering, COMPDYN, Corfu, Greece.

Lei, Z., and Qiu, C. (2000). "Neumann dynamic stochastic finite element method of vibration for structures with stochastic parameters to random excitation." Comp. Struct., 77(6), 651-657.

Li, C.-C., and Kiureghian, A. D. (1993). "Optimal discretization of random fields.” J. Eng. Mech., 10.1061/(ASCE)0733-9399(1993)119:6(1136), $1136-1154$

Lin, Y. K. (1967). Probabilistic theory of structural dynamics, McGraw Hill, New York.

Liu, W. K., Belytschko, T., and Mani, A. (1986). "Random field finiteelements." Int. J. Numer. Methods Eng., 23(10), 1831-1845.

Lucor, D., Su, C.-H., and Karniadakis, G. E. (2004). "Generalized polynomial chaos and random oscillators." Int. J. Numer. Methods Eng., 60(3), 571-596.

Matthies, H. (2007). "Uncertainty quantification with stochastic finite elements." Encyclopedia of computational mechanics.

Matthies, H. G., and Keese, A. (2005). "Galerkin methods for linear and nonlinear elliptic stochastic partial differential equations." Comput. Methods Appl. Mech. Eng., 194(12-16), 1295-1331.

Nair, P. B. (2002). "Equivalence between the combined approximations technique and Krylov subspace methods." AIAA J., 40(5), 1021-1023.

Nair, P. B., and Keane, A. J. (2002). "Stochastic reduced basis methods." AIAA J., 40(8), 1653-1664.

Najm, H. N. (2009). "Uncertainty quantification and polynomial chaos techniques in computational fluid dynamics." Annu. Rev. Fluid Mech., 41(1), 35-52.

Newmark, N. M. (1959). "A method of computation for structural dynamics." J. Engrg. Mech. Div., 85(7), 67-94.

Nickel, R. E. (1971). "On the stability of approximation operators in problems of structural dynamics." Int. J. Solids Struct., 7(3), 301-319.

Nouy, A. (2007). "A generalized spectral decomposition technique to solve a class of linear stochastic partial differential equations." Comput. Methods Appl. Mech. Eng., 196(45-48), 4521-4537.

Nouy, A. (2008). "Generalized spectral decomposition method for solving stochastic finite element equations: Invariant subspace problem and dedicated algorithms." Comput. Methods Appl. Mech. Eng., 197(51-52), $4718-4736$. 
Papadrakakis, M., and Papadopoulos, V. (1996). "Robust and efficient methods for stochastic finite element analysis using Monte Carlo simulation." Comput. Methods Appl. Mech. Eng., 134(3-4), 325-340.

Pettit, C., and Beran, P. (2006). "Spectral and multiresolution wiener expansions of oscillatory stochastic processes." J. Sound Vibrat., 294(45), 752-779.

Powell, C., and Elman, H. (2008). "Block-diagonal preconditioning for spectral stochastic finite-element systems." IMA J. Numer. Anal., 29(2), 350-375.

Pradlwarter, H. J., and Schuëller, G. I. (1997). "On advanced Monte Carlo simulation procedures in stochastic structural dynamics." Int. J. Nonlinear Mech., 32(4), 735-744.

Sachdeva, S. K., Nair, P. B., and Keane, A. J. (2006). "Hybridization of stochastic reduced basis methods with polynomial chaos expansions." Probab. Eng. Mech., 21(2), 182-192.

Sarkar, A. and Ghanem, R. (2003). "A substructure approach for the midfrequency vibration of stochastic systems." J. Acoust. Soc. Am., 113(4), 1922-1934.

Schuëller, G. I. (2001). "Computational stochastic mechanics-Recent advances." Comp. Struct., 79(22-25), 2225-2234.

Schuëller, G. I., Pradlwarter, H. J., and Bucher, C. G. (1991). "Efficient computational procedures for reliability estimates of MDOF-systems." Int. J. Non-linear Mech., 26(6), 961-974.

Shinozuka, M. (1972). "Monte Carlo solution of structural dynamics." Comp. Struct., 2(5-6), 855-874.
Vanmarcke, E. H. (1983). Random fields, MIT Press, Cambridge, MA.

Wall, F., and Bucher, C. (1987). "Sensitivity of expected exceedance rate of SDOF-system response to statistical uncertainties of loading and system parameters." Probab. Eng. Mech., 2(3), 138-146.

Wan, X. L. and Karniadakis, G. E. (2006). "Beyond Wiener-Askey expansions: Handling arbitrary PDFs.” J. Sci. Comput., 27(3), 455-464.

Xiu, D., and Karniadakis, G. E. (2002). "The Wiener-Askey polynomial chaos for stochastic differential equations." SIAM J. Sci. Comput., 24(2), 619-644.

Xiu, D., and Karniadakis, G. E. (2003a). "A new stochastic approach to transient heat conduction modeling with uncertainty." Int. J. Heat Mass Transfer, 46(24), 4681-4693.

Xiu, D., and Karniadakis, G. E. (2003b). "Modeling uncertainty in flow simulations via generalized polynomial chaos." J. Comput. Phys., 187 (1), 137-167.

Yamazaki, F., Member, A., Shinozuka, M., and Dasgupta, G. (1988). "Neumann expansion for stochastic finite element analysis." J. Eng. Mech., 10.1061/(ASCE)0733-9399(1988)114:8(1335), 1335-1354.

Yamazaki, F., and Shinozuka, M. (1988). "Digital generation of nonGaussian stochastic fields." J. Eng. Mech., 10.1061/(ASCE)07339399(1988)114:7(1183), 1183-1197.

Zhu, W. Q., Ren, Y. J., and Wu, W. Q. (1992). "Stochastic FEM based on local averages of random vector fields." J. Eng. Mech., 10.1061/(ASCE) 0733-9399(1992)118:3(496), 496-511. 


\section{AUTHOR QUERIES}

\section{AUTHOR PLEASE ANSWER ALL QUERIES}

Q: 1_Please check that ASCE Membership Grades (Member ASCE, Fellow ASCE, etc.) are provided for all authors who are members.

Q: 2_Author: Provide academic/professional title for both authors.

Q: 3_Author: Provide affiliation including department, institution (include full mailing address if the institution is not a university), city, country and postal code in affiliation 2 .

Q: 4_Author: Confirm that changes made to the sentence beginning "Various MCS..." are correct.

Q: 5_Author: Confirm that changes made to the sentence beginning "The Wiener-Hermite..." are correct.

Q: 6_In ASCE style, most variables are set in italic, and vectors and matrices in roman bold; please verify if this is correct throughout the paper.

Q: 7_Author: Please expand iid.

Q: 8_Author: Confirm that changes made to the sentence beginning "Next, the..." are correct.

Q: 9_Author: OK to change d.o.f. to DOF, as per journal style?

Q: 10_Author: Is “....in the choice of time step to be guided..." correct? Please advise.

Q: 11_Author: Confirm that "Definition 1" is correctly changed to a level 3 heading.

Q: 12_Author: Confirm that changes made to Eq. (36) are correct.

Q: 13_Author: Confirm that changes made to the formatting of Algorithm 1 do not incorrectly alter the meaning. Please also verify if "Algorithm 1: Transient Stochastic FEM with Spectral Functions" should be kept as a section heading; could this be deleted, and the algorithm begin directly after the paragraph introducing it?

Q: 14_Author: Should the Steps 4 and 5 be combined to one line?

Q: 15_Author: Should Steps 6-8 be combined into two steps? Please advise.

Q: 16_Author: Confirm that it is correct to change "N-s" to "N.s".

Q: 17_Author: Should "E" in Eq. (56) be changed to " $\mathbb{E}$ "?

Q: 18_Author: Provide city of publication in Bathe (1996).

Q: 19_Author: Provide publisher location in Bobrowski (2005).

Q: 20_Please provide the name and location of the publisher for Kundu and Adhikari (2011); if there is no "publisher," please provide the name and location of the sponsor.

Q: 21_Author: Provide page range in Kundu and Adhikari (2011).

Q: 22_Author: Provide chapter number, publisher, publisher location and page range in Matthies (2007).

Q: 23_Author: Confirm that changes made to Wan and Karniadakis (2006) are correct.

Q: 24_Author: A duplicate reference was found for Yamazaki et al. (1988a) and (1988b). One of the references was deleted and changed to Yamazaki et al. (1988). Please confirm that changes are correct.

Q: 25_Author: Confirm that changes made to Fig. 6 legend are correct.

Q: 26_Author: Please confirm that table legend added to Table 1 is correct.

Q: 27_Author: The sentence beginning "The last entry..." is unclear. Please revise.

Q: 28_Please clarify the " $F$ " character used in this section; should this be left as-is, or should it be changed to "F"? 